\title{
The combinatorics of reasonable ultrafilters
}

\author{
by \\ Saharon Shelah (Jerusalem and New Brunswick, NJ)
}

\begin{abstract}
We are interested in generalizing part of the theory of ultrafilters on $\omega$ to larger cardinals. Here we set the scene for further investigations introducing properties of ultrafilters in strong sense dual to being normal.
\end{abstract}

0. Introduction. Questions concerning ultrafilters on $\omega$ have occurred to be very stimulating for research in several subareas of set theory and topology. We hope that this success story could be repeated for ultrafilters on uncountable regular cardinals $\lambda$, particularly if $\lambda$ is strongly inaccessible. Our aim in the present paper is to introduce new properties of ultrafilters and argue that these properties could play the stimulating role that was once played by $P$-points on $\omega$.

In the long run, we plan to find generalizations of the following results:

(a) Consistently, some ultrafilters on $\omega$ are generated by $<2^{\aleph_{0}}$ sets.

(b) $P$-points are preserved by some forcing notions (see, e.g., [14, V], [11]).

(c) Consistently, there is no $P$-point.

(d) For a function $f: \omega \rightarrow \omega$ and an ultrafilter $D$ on $\omega$, let

$$
D / f:=\left\{A \subseteq \omega: f^{-1}(A) \in D\right\} ;
$$

it is an ultrafilter on $\omega$ (of course, we are interested in the cases when $D$ and $D / f$ are uniform, which in this case is the same as non-principal). By Blass and Shelah [1], consistently for any two non-principal ultrafilters $D_{1}, D_{2}$ on $\omega$ there are finite-to-one nondecreasing functions $f_{1}, f_{2}: \omega \rightarrow \omega$ such that $D_{1} / f_{1}=D_{2} / f_{2}$.

2000 Mathematics Subject Classification: Primary 03E05; Secondary 03E20.

Key words and phrases: reasonable ultrafilter, dominating family, uncountable regular cardinal.

The author acknowledges support from the United States-Israel Binational Science Foundation (Grant no. 2002323). Publication 830. 
(f) For a significant family of forcing notions built according to the scheme of creatures of [11] we may consider an appropriate filter, i.e., if $\left\langle p_{\alpha}: \alpha<\omega_{1}\right\rangle$ is $\leq^{*}$-increasing it may define an ultrafilter (see $[11, \S 5,6])$ which is not necessarily generated by $\aleph_{1}$-sets, so we may ask on this.

There are many works on normal ultrafilters; their parallel on $\omega$ are Ramsey ultrafilters. Now, every Ramsey ultrafilter on $\omega$ is a $P$-point but there are $P$-points of very different characters, e.g., $P$-point with no Ramsey ultrafilter below. Gitik [4] has investigated generalizations of $P$-points for normal ultrafilters. But this paper goes in a different direction (which up to recently I have not considered to be fruitful) and we restrict our attention to ultrafilters which are very non-normal - the weakly reasonable ultrafilters. What is a weakly reasonable ultrafilter on $\lambda$ ? It is a uniform ultrafilter on a regular cardinal $\lambda$ which does not contain some club of $\lambda$ and such that this property is preserved if we divide it by a non-decreasing $f: \lambda \rightarrow \lambda$ with unbounded range (see Definition 1.4 below).

We also want that our ultrafilters generalize $P$-points on $\omega$ and in the second section we introduce reasonable and very reasonable ultrafilters. The property defining $P$-points is that countable families of sets from the ultrafilter have pseudo-intersections in the ultrafilter. We modify this property so that we involve some description of how the ultrafilter considered is generated, and we postulate that the generating systems are suitably directed. This is a replacement for the existence of pseudo-intersections and it is the essence of Definition 2.5(4,5). The third section shows that the number of generating systems (of our type) for somewhat reasonable ultrafilters cannot be too small. We conclude the paper with a section listing open problems and describing further research.

Notation. Our notation is rather standard and compatible with that of classical textbooks (like Jech [5]). In forcing we keep the older convention that a stronger condition is the larger one. (However, in the present paper we use forcing notions only for combinatorial constructions and almost every mention of forcing just means that we are dealing with a transitive reflexive relation $\mathbb{P}=\left(\mathbb{P}, \leq_{\mathbb{P}}\right)$.)

(1) Ordinal numbers will be denoted by lower case initial letters of the Greek alphabet $(\alpha, \beta, \gamma, \delta, \ldots)$ and also by $i, j$ (with possible suband superscripts).

(2) Cardinal numbers will be called $\kappa, \lambda, \mu ; \lambda$ will always be assumed to be a regular uncountable cardinal (we may forget to mention it).

(3) $D, \mathcal{U}$ will denote filters on $\lambda$, and $G, G^{*}, G_{\ell}^{*}$ will be subsets of specific partial orders used to generate filters on $\lambda$. 
(4) A bar above a letter denotes that the object considered is a sequence; usually $\bar{X}$ will be $\left\langle X_{i}: i\langle\zeta\rangle\right.$, where $\zeta$ is the lengthlh $(\bar{X})$ of $\bar{X}$. Sometimes our sequences will be indexed by a set of ordinals, say $S \subseteq \lambda$, and then $\bar{X}$ will typically be $\left\langle X_{\delta}: \delta \in S\right\rangle$.

Definition 0.1. A dominating family in ${ }^{\lambda} \lambda$ is a family $\mathcal{F} \subseteq{ }^{\lambda} \lambda$ such that

$$
\left(\forall g \in{ }^{\lambda} \lambda\right)(\exists f \in \mathcal{F})(\exists \alpha<\lambda)(\forall \beta>\alpha)(g(\beta)<f(\beta)) .
$$

The $\lambda$-dominating number $\mathfrak{d}_{\lambda}$ is defined as

$$
\mathfrak{d}_{\lambda}=\min \left\{\|\mathcal{F}\|: \mathcal{F} \subseteq{ }^{\lambda} \lambda \text { is a dominating family in }{ }^{\lambda} \lambda\right\} .
$$

A club-dominating family in ${ }^{\lambda} \lambda$ is a family $\mathcal{F} \subseteq{ }^{\lambda} \lambda$ such that

$$
\left(\forall g \in{ }^{\lambda} \lambda\right)(\exists f \in \mathcal{F})(\{\beta<\lambda: g(\beta) \geq f(\beta)\} \text { is non-stationary in } \lambda) .
$$

The $\operatorname{cl}(\lambda)$-dominating number $\mathfrak{d}_{\mathrm{cl}}(\lambda)$ is defined as

$$
\mathfrak{d}_{\mathrm{cl}}(\lambda)=\min \left\{\|\mathcal{F}\|: \mathcal{F} \subseteq{ }^{\lambda} \lambda \text { is a } \operatorname{cl}(\lambda) \text {-dominating family in }{ }^{\lambda} \lambda\right\} .
$$

On $\mathfrak{d}_{\lambda}, \mathfrak{d}_{\mathrm{cl}}(\lambda)$ see, e.g., in Cummings and Shelah [2].

Acknowledgments. I thank Tomek Bartoszyński and Andrzej Rosłanowski for stimulating discussions, and the anonymous referee for valuable comments.

1. Weakly reasonable ultrafilters. In Definition 1.4(1) we formulate the main property of ultrafilters on $\lambda$ which is of interest to us: being a weakly reasonable ultrafilter. In the spectrum of all ultrafilters, weakly reasonable ultrafilters are at the opposite end to normal ultrafilters. We show that there exist (in ZFC) weakly reasonable ultrafilters (see 1.10) and we also give some properties of such ultrafilters.

Definition 1.1. For a cardinal $\lambda$,

(a) $\operatorname{ulf}(\lambda)$ is the set of all ultrafilters on $\lambda$,

(b) $\operatorname{uuf}(\lambda)$ is the family of all uniform ultrafilters on $\lambda$,

(c) if $D$ is an ultrafilter on $\lambda$ and $f \in{ }^{\lambda} \lambda$, then

$$
D / f:=\left\{A \subseteq \lambda: f^{-1}(A) \in D\right\} .
$$

Let us note that in the literature $D / f$ is also denoted by $f(D)$ or $f_{*}(D)$ and it is called the image or the projection of the ultrafilter $D$ under $f$. We use the quotient notation and terminology because we will deal mostly with $D / C$, where:

Definition 1.2. Assume $D$ is an ultrafilter on $\lambda$.

(1) If $E$ is an equivalence relation on $\lambda$, then $f_{E} \in{ }^{\lambda} \lambda$ is defined by

$$
f_{E}(\alpha)=\operatorname{otp}(\{\beta<\alpha: \beta=\min (\beta / E)<\min (\alpha / E)\}),
$$


and $D / E$ is $D / f_{E}$. (Here, $\alpha / E$ stands for the $E$-equivalence class of $\alpha$.)

(2) For a club $C$ of $\lambda$ let $E_{C}$ be the following equivalence relation on $\lambda$ :

$$
\alpha E_{C} \beta \quad \text { iff } \quad(\forall \gamma \in C)(\alpha<\gamma \Leftrightarrow \beta<\gamma)
$$

(so $E_{C}$ is the equivalence relation determined by the partition of $\lambda$ into intervals $[\xi, \zeta)$ for consecutive members $\xi<\zeta$ of $C \cup\{0\})$. Let $D / C$ be $D / E_{C}$.

(3) $\mathcal{F}_{\lambda}$ is the family of all non-decreasing unbounded functions from $\lambda$ to $\lambda$.

ObSeRVATion 1.3. Assume that $\lambda$ is a regular cardinal and $D \in \operatorname{ulf}(\lambda)$.

(1) If $f: \lambda \rightarrow \lambda$, then $D / f \in \operatorname{ulf}(\lambda)$.

(2) If $f \in \mathcal{F}_{\lambda}$ and $D$ is uniform, then also $D / f$ is a uniform ultrafilter on $\lambda$.

(3) If $C$ is a club of $\lambda$ and $\left\langle\delta_{\xi}: \xi<\lambda\right\rangle$ is the increasing enumeration of $C \cup\{0\}$, then for a set $A \subseteq \lambda$,

$$
A \in D / C \quad \text { if and only if } \bigcup\left\{\left[\delta_{\xi}, \delta_{\xi+1}\right): \xi \in A\right\} \in D .
$$

DeFinition 1.4. Let $D$ be a uniform ultrafilter on $\lambda$.

(1) We say that $D$ is weakly reasonable if for every $f \in \mathcal{F}_{\lambda}$ there is a club $C$ of $\lambda$ such that

$$
\bigcup\{[\delta, \delta+f(\delta)): \delta \in C\} \notin D .
$$

(2) We define a game $\partial_{D}$ between two players, Odd and Even, as follows. A play of $\partial_{D}$ lasts $\lambda$ steps and during a play an increasing continuous sequence $\bar{\alpha}=\left\langle\alpha_{i}: i\langle\lambda\rangle \subseteq \lambda\right.$ is constructed. The terms of $\bar{\alpha}$ are chosen successively by the two players so that Even chooses the $\alpha_{i}$ for even $i$ (including limit stages $i$ where she has no free choice) and Odd chooses $\alpha_{i}$ for odd $i$.

Even wins the play if and only if

$$
\bigcup\left\{\left[\alpha_{2 i+1}, \alpha_{2 i+2}\right): i<\lambda\right\} \in D .
$$

ObSERVATION 1.5. Let $D \in \operatorname{uuf}(\lambda)$. Then the following conditions are equivalent:

(A) D is weakly reasonable,

(B) for every increasing continuous sequence $\left\langle\delta_{\xi}: \xi<\lambda\right\rangle \subseteq \lambda$ there is a club $C^{*}$ of $\lambda$ such that

$$
\bigcup\left\{\left[\delta_{\xi}, \delta_{\xi+1}\right): \xi \in C^{*}\right\} \notin D
$$

(C) for every club $C$ of $\lambda$ the quotient $D / C$ does not extend the filter generated by clubs of $\lambda$. 
Proposition 1.6. Assume $D \in \operatorname{uuf}(\lambda)$.

(1) If $\lambda$ is strongly inaccessible and Odd has a winning strategy in $\partial_{D}$, then $D$ is not weakly reasonable.

(2) If $D$ is not weakly reasonable, then Odd has a winning strategy in $\partial_{D}$.

(3) In (1), instead of " $\lambda$ is strongly inaccessible", it suffices to assume $\diamond_{\lambda}^{*}$.

Proof. (1) Suppose towards a contradiction that $\lambda$ is strongly inaccessible, Odd has a winning strategy st in the game $\partial_{D}$ but $D$ is weakly reasonable. By induction on $\varepsilon<\lambda$ choose an increasing continuous sequence $\left\langle N_{\varepsilon}: \varepsilon<\lambda\right\rangle$ of elementary submodels of $\mathcal{H}\left(\lambda^{++}\right)$so that for each $\varepsilon$ :

(a) $N_{\varepsilon} \prec\left(\mathcal{H}\left(\lambda^{++}\right), \in,<^{*}\right),\left\|N_{\varepsilon}\right\|<\lambda, N_{\varepsilon} \cap \lambda \in \lambda$,

(b) ${ }^{\varepsilon} N_{\varepsilon+1} \subseteq N_{\varepsilon+1}$,

(c) $\left\langle N_{\zeta}: \zeta \leq \varepsilon\right\rangle \in N_{\varepsilon+1}$,

(d) st, $\lambda, D$ belong to $N_{0}$.

Let $\delta_{\varepsilon}=N_{\varepsilon} \cap \lambda$ (for $\varepsilon<\lambda$ ). Then $\left\langle\delta_{\varepsilon}: \varepsilon<\lambda\right\rangle$ is an increasing continuous sequence of limit ordinals. Let $f(\alpha)=\delta_{\alpha+1}$ for $\alpha<\lambda$, so $f \in \mathcal{F}_{\lambda}$.

Since $D$ is a weakly reasonable ultrafilter, there is a club $C$ of $\lambda$ such that

$$
\bigcup\{[\delta, \delta+f(\delta)): \delta \in C\} \notin D .
$$

Let

$$
C^{*}=\left\{\varepsilon \in C: \varepsilon=\delta_{\varepsilon} \text { is a limit ordinal }\right\}
$$

(it is a club of $\lambda$ ). Then for $\varepsilon \in C^{*}$ we have $\left[\delta_{\varepsilon}, \delta_{\varepsilon+1}\right) \subseteq[\varepsilon, \varepsilon+f(\varepsilon))$ and hence

$$
\bigcup\left\{\left[\delta_{\varepsilon}, \delta_{\varepsilon+1}\right): \varepsilon \in C^{*}\right\} \notin D .
$$

Let us define a strategy $\mathbf{s t}^{\prime}$ for Even in the game $\partial_{D}$ as follows. For an even ordinal $i<\lambda$, in the $i$ th move of a play, if $\left\langle\alpha_{j}: j<i\right\rangle$ has been played so far then Even plays

$$
\alpha_{i}= \begin{cases}\sup \left\{\alpha_{j}: j<i\right\} & \text { if } i \text { is limit, } \\ \min \left\{\varepsilon \in C^{*}:(\forall j<i)\left(\alpha_{j}<\varepsilon\right)\right\} & \text { otherwise. }\end{cases}
$$

Now consider a play $\left\langle\alpha_{i}: i<\lambda\right\rangle$ in which Even uses the strategy st $^{\prime}$ and Odd plays according to st. Then for each $i<\lambda$ we have $\alpha_{2 i} \in C^{*}$ and thus $\alpha_{2 i}=\delta_{\alpha_{2 i}} \in N_{\alpha_{2 i}+1}$, and also $\left\{\alpha_{j}: j<2 i\right\} \subseteq \alpha_{2 i} \subseteq N_{\alpha_{2 i}+1}$. Since the model $N_{\alpha_{2 i}+1}$ is closed under forming sequences of length $\alpha_{2 i}+1$ (by (b)), we conclude that $\left\langle\alpha_{j}: j \leq 2 i\right\rangle \in N_{\alpha_{2 i}+1}$. Since st $\in N_{0} \prec N_{\alpha_{2 i}+1}$, clearly $\alpha_{2 i+1} \in N_{\alpha_{2 i}+1} \cap \lambda$ and therefore $\alpha_{2 i+1}<\delta_{\alpha_{2 i}+1}$. Hence

$$
\begin{aligned}
\bigcup\left\{\left[\alpha_{2 i}, \alpha_{2 i+1}\right): i<\lambda\right\} & \subseteq \bigcup\left\{\left[\delta_{\alpha_{2 i}}, \delta_{\alpha_{2 i}+1}\right): i<\lambda\right\} \\
& \subseteq \bigcup\left\{\left[\delta_{\varepsilon}, \delta_{\varepsilon+1}\right): \varepsilon \in C^{*}\right\} \notin D .
\end{aligned}
$$


But st is a winning strategy for Odd, so he wins the play and

$$
\bigcup\left\{\left[\alpha_{2 i+1}, \alpha_{2 i+2}\right): i<\lambda\right\} \notin D,
$$

a contradiction.

(2) Suppose that $D \in \operatorname{uuf}(\lambda)$ is not weakly reasonable. Then we may find $f \in \mathcal{F}_{\lambda}$ such that for every club $C$ of $\lambda$ we have

$$
\bigcup\{[\delta, \delta+f(\delta)): \delta \in C\} \in D .
$$

Let st be a strategy of Odd in $\partial_{D}$ which instructs him to play as follows. For an odd ordinal $i=i_{0}+1<\lambda$, in the $i$ th move of a play, if $\left\langle\alpha_{j}: j \leq i_{0}\right\rangle$ has been played so far, then Odd plays $\alpha_{i}=\alpha_{i_{0}}+f\left(\alpha_{i_{0}}\right)+1$.

We claim that st is a winning strategy for Odd (in $\partial_{D}$ ). To this end suppose that $\left\langle\alpha_{j}: j\langle\lambda\rangle \subseteq \lambda\right.$ is a result of a play of $\partial_{D}$ in which Odd uses the strategy st. Let $C^{\prime}=\left\{\alpha_{i}: i<\lambda\right.$ is limit $\}$; it is a club of $\lambda$, so by the choice of $f$ we have

$$
\bigcup\left\{[\delta, \delta+f(\delta)): \delta \in C^{\prime}\right\} \in D
$$

Since $\bigcup\left\{[\delta, \delta+f(\delta)): \delta \in C^{\prime}\right\} \subseteq \bigcup\left\{\left[\alpha_{2 i}, \alpha_{\alpha_{2 i+1}}\right): i<\lambda\right\}$ we may now conclude that Odd indeed wins the play.

REMARK 1.7. Let us note that some assumptions on $\lambda$ in 1.6(1) are needed. This will be shown in the subsequent paper of Rosłanowski and Shelah [7].

LEMMA 1.8. Suppose that $\lambda$ is a regular uncountable cardinal, $D \in$ $\operatorname{uuf}(\lambda)$ is a weakly reasonable ultrafilter and $\left\langle\beta_{i}: i<\lambda\right\rangle$ is an increasing continuous sequence of ordinals below $\lambda$. Then there is an increasing continuous sequence $\left\langle\delta_{\xi}: \xi\langle\lambda\rangle \subseteq \lambda\right.$ consisting of limit ordinals and such that

$$
\bigcup\left\{\left[\beta_{\delta_{2 \xi+1}}, \beta_{\delta_{2 \xi+2}}\right): \xi<\lambda\right\} \in D .
$$

Proof. It follows from 1.5 that we may find a club $C^{*}$ of $\lambda$ such that all members of $C^{*}$ are limit ordinals and $\bigcup\left\{\left[\beta_{\xi}, \beta_{\xi+1}\right): \xi \in C^{*}\right\} \notin D$. Let $C^{+}=C^{*} \cup\left\{\xi+1: \xi \in C^{*}\right\}$ (clearly it is a club of $\lambda$ ) and let $\left\langle\delta_{\xi}: \xi<\lambda\right\rangle$ be the increasing enumeration of $C^{+}$. Note that $C^{*}=\left\{\delta_{\xi}: \xi<\lambda\right.$ is even $\}$ and, for an even ordinal $\xi<\lambda, \delta_{\xi+1}=\delta_{\xi}+1$. Hence

$$
\begin{aligned}
\bigcup\left\{\left[\beta_{\delta_{\xi}}, \beta_{\delta_{\xi+1}}\right): \xi<\lambda \text { is even }\right\} & =\bigcup\left\{\left[\beta_{\delta_{\xi}}, \beta_{\delta_{\xi}+1}\right): \xi<\lambda \text { is even }\right\} \\
& =\bigcup\left\{\left[\beta_{\zeta}, \beta_{\zeta+1}\right): \zeta \in C^{*}\right\} \notin D .
\end{aligned}
$$

Consequently, $\bigcup\left\{\left[\beta_{\delta_{\xi}}, \beta_{\delta_{\xi+1}}\right): \xi<\lambda\right.$ is odd $\} \in D$.

THEOREM 1.9. If $\lambda$ is a regular uncountable cardinal and $D \in \operatorname{uuf}(\lambda)$ is weakly reasonable, then $D$ is a regular ultrafilter. 
Proof. Using Lemma 1.8 we may choose by induction on $\varepsilon<\lambda$ a sequence $\left\langle\bar{\delta}^{\varepsilon}: \varepsilon<\lambda\right\rangle$ so that

(a) $\bar{\delta}^{\varepsilon}=\left\langle\delta_{i}^{\varepsilon}: i<\lambda\right\rangle$ is an increasing continuous sequence of nonsuccessor ordinals below $\lambda, \delta_{0}^{\varepsilon}=0$,

(b) the set $A_{\varepsilon}:=\bigcup\left\{\left[\delta_{2 i+1}^{\varepsilon}, \delta_{2 i+2}^{\varepsilon}\right): i<\lambda\right\}$ belongs to $D$,

(c) if $\zeta<\varepsilon, i<\lambda$, then $\delta_{i}^{\varepsilon} \in\left\{\delta_{j}^{\zeta}: j<\lambda\right.$ is a limit ordinal or zero $\}$.

For $\varepsilon<\lambda$ let $f_{\varepsilon}: A_{\varepsilon} \rightarrow \lambda$ be such that

$$
\alpha \in\left[\delta_{2 i+1}^{\varepsilon}, \delta_{2 i+2}^{\varepsilon}\right) \Rightarrow f_{\varepsilon}(\alpha)=\delta_{2 i+1}^{\varepsilon} .
$$

Note that

$(\otimes)$ if $\zeta<\varepsilon<\lambda, \alpha \in A_{\zeta} \cap A_{\varepsilon}$, then $f_{\varepsilon}(\alpha)<f_{\zeta}(\alpha)$.

[Why? Let $f_{\zeta}(\alpha)=\delta_{2 i+1}^{\zeta}$ (so $\alpha \in\left[\delta_{2 i+1}^{\zeta}, \delta_{2 i+2}^{\zeta}\right)$ ). It follows from (c) that $f_{\varepsilon}(\alpha) \in\left\{\delta_{j}^{\zeta}: j<\lambda\right.$ is a limit ordinal or zero $\}$ and hence (as also $f_{\varepsilon}(\alpha) \leq \alpha$ ) we may conclude that $f_{\varepsilon}(\alpha)<f_{\zeta}(\alpha)$.]

For $\alpha<\lambda$, let $w_{\alpha}=\left\{\varepsilon<\lambda: \alpha \in A_{\varepsilon}\right\}$. It follows from ( $\left.\otimes\right)$ that (for every $\alpha<\lambda)$ the sequence $\left\langle f_{\varepsilon}(\alpha): \varepsilon \in w_{\alpha}\right\rangle$ is strictly decreasing, so necessarily each $w_{\alpha}$ is finite. Since $A_{\varepsilon} \in D$ for each $\varepsilon<\lambda$ (by (b)), we have shown the regularity of $D$.

THEOREM 1.10. Let $\lambda>\aleph_{0}$ be a regular cardinal. Then there is a uniform weakly reasonable ultrafilter $D$ on $\lambda$.

Proof. Let $\left\{f_{\varepsilon}: \varepsilon<\mathfrak{d}_{\lambda}\right\} \subseteq{ }^{\lambda} \lambda$ be a dominating family and for $\varepsilon<\mathfrak{d}_{\lambda}$ let $C_{\varepsilon}$ be a club of $\lambda$ such that members of $C_{\varepsilon}$ are limit ordinals and

$$
\left(\forall \delta \in C_{\varepsilon}\right)(\forall \alpha<\delta)\left(f_{\varepsilon}(\alpha)<\delta\right) .
$$

Let $\left\langle\alpha_{\varepsilon, i}: i<\lambda\right\rangle$ be the increasing enumeration of $C_{\varepsilon}$.

By induction on $\varepsilon$ we will choose sets $E_{\varepsilon}, A_{\varepsilon}$ so that for each $\varepsilon<\mathfrak{d}_{\lambda}$ :

(a) $A_{\varepsilon}$ is an unbounded subset of $\lambda$ and $E_{\varepsilon} \subseteq C_{\varepsilon}$ is a club of $\lambda$,

(b) $A_{\varepsilon} \cap \bigcup\left\{\left[\alpha_{\varepsilon, \gamma}, \alpha_{\varepsilon, \gamma+1}\right): \gamma \in E_{\varepsilon}\right\}=\emptyset$,

(c) if $n<\omega$ and $\zeta_{0}<\cdots<\zeta_{n-1}<\varepsilon$, then $\left\|A_{\varepsilon} \cap \bigcap_{i<n} A_{\zeta_{i}}\right\|=\lambda$.

So suppose that we have chosen $A_{\zeta}, E_{\zeta}$ for $\zeta<\varepsilon<\mathfrak{d}_{\lambda}$ so that the respective reformulations of (a)-(c) hold true. For a finite sequence $\bar{\zeta}=\left\langle\zeta_{i}: i<n\right\rangle$ of ordinals below $\varepsilon$ let $A^{\bar{\zeta}}=\bigcap_{i<n} A_{\zeta_{i}}$ (note that $\left\|A^{\bar{\zeta}}\right\|=\lambda$ by the demand in $(\mathrm{c}))$. Let $g_{\bar{\zeta}}^{\varepsilon} \in{ }^{\lambda} \lambda$ be such that

$(\oplus)$ if $\alpha_{\varepsilon, i} \leq \alpha<\alpha_{\varepsilon, i+1}$, then $g_{\bar{\zeta}}^{\varepsilon}(\alpha)=\min \left\{\delta>\alpha_{\varepsilon, i+1}:\left[\alpha_{\varepsilon, i+1}, \delta\right) \cap A^{\bar{\zeta}}\right.$ $\neq \emptyset\}$.

The family $\left\{g_{\bar{\zeta}}^{\varepsilon}: \bar{\zeta} \in{ }^{\omega>} \varepsilon\right\}$ is a subset of ${ }^{\lambda} \lambda$ of cardinality $\leq|\varepsilon|+\aleph_{0}<\mathfrak{d}_{\lambda}$, so it cannot be a dominating family. Therefore we may pick a function $h_{\varepsilon} \in{ }^{\lambda} \lambda$ 
such that

$$
\left(\forall \bar{\zeta} \in{ }^{\omega>} \varepsilon\right)\left(\exists^{\lambda} \alpha<\lambda\right)\left(g_{\bar{\zeta}}^{\varepsilon}(\alpha)<h_{\varepsilon}(\alpha)\right)
$$

Put

$$
\begin{aligned}
& E_{\varepsilon}=\left\{\delta<\lambda: \delta=\alpha_{\varepsilon, \delta} \text { is a limit ordinal and }(\forall \alpha<\delta)\left(h_{\varepsilon}(\alpha)<\delta\right)\right\} \\
& A_{\varepsilon}=\bigcup\left\{\left[\alpha_{\varepsilon, \gamma+1}, \alpha_{\varepsilon, \delta}\right): \gamma<\delta \text { are successive members of } E_{\varepsilon}\right\} .
\end{aligned}
$$

It should be clear that $E_{\varepsilon}, A_{\varepsilon}$ satisfy demands (a), (b).

Let us argue that also condition (c) holds true. Let $\bar{\zeta} \in{ }^{\omega>} \varepsilon$ and we shall prove that $A_{\varepsilon} \cap A^{\bar{\zeta}}$ is unbounded in $\lambda$. By the choice of $h_{\varepsilon}$, the set $B=$ $\left\{\alpha<\lambda: g_{\bar{\zeta}}^{\varepsilon}(\alpha)<h_{\varepsilon}(\alpha)\right\}$ is of cardinality $\lambda$. Let us fix $\alpha \in B$ for a moment and let $i<\lambda$ be such that $\alpha_{\varepsilon, i} \leq \alpha<\alpha_{\varepsilon, i+1}$. Let $\sup \left(E_{\varepsilon} \cap \alpha_{\varepsilon, i+1}\right)=\gamma=\alpha_{\varepsilon, \gamma}$ and $\min \left(E_{\varepsilon} \backslash \alpha_{\varepsilon, \gamma+1}\right)=\delta=\alpha_{\varepsilon, \delta}$. Then $\gamma, \delta$ are successive members of $E_{\varepsilon}$ and

$$
\gamma \leq \alpha_{\varepsilon, i} \leq \alpha<\alpha_{\varepsilon, i+1}<\delta
$$

Hence (by the definition of $E_{\varepsilon}$ and since $\alpha \in B$ ) we get

$$
\left[\alpha_{\varepsilon, i+1}, g_{\bar{\zeta}}^{\varepsilon}(\alpha)\right) \subseteq\left[\alpha_{\varepsilon, i+1}, h_{\varepsilon}(\alpha)\right) \subseteq\left[\alpha_{\varepsilon, \gamma+1}, \alpha_{\varepsilon, \delta}\right) \subseteq A_{\varepsilon}
$$

It follows from $(\oplus)$ that $\left[\alpha_{\varepsilon, i+1}, g_{\bar{\zeta}}^{\varepsilon}(\alpha)\right) \cap A^{\bar{\zeta}} \neq \emptyset$, and so $A_{\varepsilon} \cap A^{\bar{\zeta}} \backslash \alpha \neq \emptyset$. Since $\|B\|=\lambda$ we may now easily conclude that $\left\|A_{\varepsilon} \cap A^{\bar{\zeta}}\right\|=\lambda$, showing that $A_{\varepsilon}, E_{\varepsilon}$ are as required.

After the construction is carried out (and we have the sequence $\left\langle E_{\varepsilon}, A_{\varepsilon}\right.$ : $\left.\varepsilon\left\langle\mathfrak{d}_{\lambda}\right\rangle\right)$ we may find a uniform ultrafilter $D$ on $\lambda$ such that $\left\{A_{\varepsilon}: \varepsilon<\mathfrak{d}_{\lambda}\right\} \subseteq$ $D$ (remember the demand in (c)). We claim that $D$ is weakly reasonable. To this end suppose that $C$ is a club of $\lambda$ and $\left\langle\delta_{\xi}: \xi\langle\lambda\rangle \subseteq \lambda\right.$ is the increasing enumeration of $C$. By the choice of $f_{\varepsilon}, C_{\varepsilon}$ (for $\varepsilon<\mathfrak{d}_{\lambda}$ ) we may find $\varepsilon<\mathfrak{d}_{\lambda}$ and $j_{0}<\lambda$ such that

$$
\left(\forall i \geq j_{0}\right)\left(\left\|\left[\alpha_{\varepsilon, i}, \alpha_{\varepsilon, i+1}\right) \cap C\right\|>2\right) .
$$

Let

$$
C^{*}=\left\{\gamma \in E_{\varepsilon} \cap C \backslash j_{0}: \gamma=\alpha_{\varepsilon, \gamma}=\delta_{\gamma} \text { is a limit ordinal }\right\}
$$

(it is a club of $\lambda$ ). Since for $\gamma \in C^{*}$ we have $\alpha_{\varepsilon, \gamma}=\delta_{\gamma}<\delta_{\gamma+1}<\alpha_{\varepsilon, \gamma+1}$ we may easily conclude from (b) that

$$
\bigcup\left\{\left[\delta_{\gamma}, \delta_{\gamma+1}\right): \gamma \in C^{*}\right\} \notin D
$$

completing the proof (remember 1.5).

2. More reasonable ultrafilters. In this section we propose a property of ultrafilters stronger than being weakly reasonable (see Definition $2.5(5))$. We believe that the notion of very reasonable ultrafilters is the right 
re-interpretation of being a $P$-point in the setting of "very non-normal ultrafilters" on an uncountable regular cardinal $\lambda$. We start by describing a forcing notion $\mathbb{Q}_{\lambda}^{1}$ which motivated our choice of generating systems of 2.5 .

As before, $\lambda$ is always assumed to be an uncountable regular cardinal.

Definition 2.1. We define a forcing notion $\mathbb{Q}_{\lambda}^{1}$ as follows. A condition in $\mathbb{Q}_{\lambda}^{1}$ is a tuple $p=\left(\gamma^{p}, C^{p},\left\langle Z_{\delta}^{p}: \delta \in C^{p}\right\rangle,\left\langle d_{\delta}^{p}: \delta \in C^{p}\right\rangle\right)$ such that

(i) $\gamma^{p}<\lambda, C^{p}$ a club of $\lambda$ consisting of limit ordinals only, and for $\delta \in C^{p}$

(ii) $Z_{\delta}^{p}=\left[\delta, \min \left(C^{p} \backslash(\delta+1)\right)\right)$,

(iii) $d_{\delta}^{p} \subseteq \mathcal{P}\left(Z_{\delta}^{p}\right)$ is a proper ultrafilter on $Z_{\delta}^{p}$.

The order $\leq_{\mathbb{Q}_{\lambda}^{1}}=\leq$ of $\mathbb{Q}_{\lambda}^{1}$ is given by $p \leq_{\mathbb{Q}_{\lambda}^{1}} q$ if and only if

(a) $\gamma^{p} \leq \gamma^{q}, C^{p} \cap \gamma^{p} \subseteq C^{q} \subseteq C^{p}$,

(b) if $\delta<\varepsilon$ are successive members of $C^{q}\left(\right.$ so $Z_{\delta}^{q}=[\delta, \varepsilon)$ ), then

$$
\left(\forall A \in d_{\delta}^{q}\right)\left(\exists \zeta \in C^{p} \cap[\delta, \varepsilon)\right)\left(A \cap Z_{\zeta}^{p} \in d_{\zeta}^{p}\right) .
$$

REMARK 2.2. The forcing notion $\mathbb{Q}_{\lambda}^{1}$ can be represented according to the framework of $[10, \S$ B.5].

Proposition 2.3.

(1) $\mathbb{Q}_{\lambda}^{1}$ is a partial order, $\left\|\mathbb{Q}_{\lambda}^{1}\right\|=2^{2^{<\lambda}}$.

(2) If $p, q \in \mathbb{Q}_{\lambda}^{1}, p \leq q, \delta<\varepsilon$ are two successive members of $C^{p}$, and $\delta, \varepsilon \in C^{q}$, then $Z_{\delta}^{q}=Z_{\delta}^{p}$ and $d_{\delta}^{q}=d_{\delta}^{p}$.

(3) $\mathbb{Q}_{\lambda}^{1}$ is $(<\lambda)$-complete (so it does not add bounded subsets of $\lambda$ ).

(4) If $p \in \mathbb{Q}_{\lambda}^{1}, A \subseteq \lambda$, then there is a condition $q \in \mathbb{Q}_{\lambda}^{1}$ stronger than $p$ and such that either

$$
\left(\forall \delta \in C^{q} \backslash \gamma^{p}\right)\left(A \cap Z_{\delta}^{q} \in d_{\delta}^{q}\right) \quad \text { or } \quad\left(\forall \delta \in C^{q} \backslash \gamma^{p}\right)\left(A \cap Z_{\delta}^{q} \notin d_{\delta}^{q}\right) .
$$

Proof. (1), (2) Straightforward.

(3) Assume that $\delta<\lambda$ is a limit ordinal and a sequence $\left\langle p_{i}: i<\delta\right\rangle \subseteq \mathbb{Q}_{\lambda}^{1}$ is $\leq \mathbb{Q}_{\lambda}^{1}$-increasing. Let $E$ be a uniform ultrafilter on $\delta$. Let us put:

- $\gamma=\sup \left\{\gamma^{p_{i}}: i<\delta\right\}, C=\bigcap_{i<\delta} C^{p_{i}}$, and for $\alpha \in C$ let

- $Z_{\alpha}=[\alpha, \min (C \backslash(\alpha+1)))$ and

- $d_{\alpha}=\left\{A \subseteq Z_{\alpha}:\left\{i<\delta: A \cap Z_{\alpha}^{p_{i}} \in d_{\alpha}^{p_{i}}\right\} \in E\right\}$.

It is easy to check that $p=\left(\gamma, C,\left\langle Z_{\alpha}: \alpha \in C\right\rangle,\left\langle d_{\alpha}: \alpha \in C\right\rangle\right)$ belongs to $\mathbb{Q}_{\lambda}^{1}$ and that it is a condition stronger than all $p_{i}$ (for $i<\delta$ ).

(4) Let $p \in \mathbb{Q}_{\lambda}^{1}$ and $A \subseteq \lambda$. Just for simplicity we may assume that $\gamma^{p} \in C^{p}$ (as we may always increase $\gamma^{p}$ ). Put

$$
Y:=\left\{\alpha \in C^{p}: A \cap Z_{\alpha}^{p} \in d_{\alpha}^{p}\right\}
$$

and consider two cases. 
CAsE 1: $Y$ is unbounded in $\lambda$. Then we may choose an increasing continuous sequence $\left\langle\delta_{i}: i<\lambda\right\rangle \subseteq C^{p}$ such that $\delta_{0}=\gamma^{p}$ and $(\forall i<\lambda)\left(\left[\delta_{i}, \delta_{i+1}\right) \cap\right.$ $Y \neq \emptyset)$. Put

- $\gamma=\gamma^{p}, C=\left\{\delta_{i}: i<\lambda\right\} \cup\left(C^{p} \cap \gamma^{p}\right)$,

- if $\alpha \in C^{p} \cap \gamma^{p}$, then $Z_{\alpha}=Z_{\alpha}^{p}$ and $d_{\alpha}=d_{\alpha}^{p}$,

- if $\alpha=\delta_{i}, i<\lambda$, then $Z_{\alpha}=\left[\delta_{i}, \delta_{i+1}\right)$ and

$$
d_{\alpha}=\left\{B \subseteq Z_{\alpha}: B \cap Z_{\min (Y \backslash \alpha)}^{p} \in d_{\min (Y \backslash \alpha)}^{p}\right\} .
$$

It is straightforward to verify that $q=\left(\gamma, C,\left\langle Z_{\alpha}: \alpha \in C\right\rangle,\left\langle d_{\alpha}: \alpha \in C\right\rangle\right)$ $\in \mathbb{Q}_{\lambda}^{1}$ is a condition stronger than $p$ and it is also clear that $\left(\forall \alpha \in C \backslash \gamma^{p}\right)$ $\left(A \cap Z_{\alpha} \in d_{\alpha}\right)$.

CAsE 2: $Y$ is bounded in $\lambda$. Then the set $\lambda \backslash Y$ is unbounded, so we may apply the construction of $q$ from Case 1 replacing $Y$ by its complement $\lambda \backslash Y$. It should be clear that the condition $q$ we then get satisfies $(\forall \alpha \in$ $\left.C^{q} \backslash \gamma^{p}\right)\left(A \cap Z_{\alpha}^{q} \notin d_{\alpha}^{q}\right)$.

REMARK 2.4. The following discussion presents our motivations for the definitions and concepts presented later in this section.

Suppose that $G \subseteq \mathbb{Q}_{\lambda}^{1}$ is a generic filter over $\mathbf{V}$. In $\mathbf{V}[G]$ we define $C=\bigcup\left\{C^{p} \cap \gamma^{p}: p \in G\right\}$ and for $\alpha \in C$ we let $d_{\alpha}=d_{\alpha}^{p}$ for some (equivalently: all) $p \in G$ such that $\alpha<\gamma^{p}$ and $C^{p} \cap\left(\alpha, \gamma^{p}\right) \neq \emptyset$. Then $C$ is a club of $\lambda$ and (for $\alpha \in C) d_{\alpha}$ is an ultrafilter on $[\alpha, \min (C \backslash(\alpha+1))$ ). Let

$$
D=\left\{A \in \mathcal{P}(\lambda)^{\mathbf{V}}:(\exists \varepsilon<\lambda)(\forall \alpha>\varepsilon)\left(A \cap[\alpha, \min (C \backslash(\alpha+1))) \in d_{\alpha}\right)\right\} .
$$

It follows from 2.3(4) that $D$ is an ultrafilter on the Boolean algebra $\mathcal{P}(\lambda)^{\mathbf{V}}$.

Let $\underset{\sim}{D}$ be a $\mathbb{Q}_{\lambda}^{1}$-name for the $D$ defined as above. Note that if $p \in \mathbb{Q}_{\lambda}^{1}$, $A \subseteq \lambda$ and $(\exists \varepsilon<\lambda)\left(\forall \delta \in C^{p} \backslash \varepsilon\right)\left(A \cap Z_{\delta}^{p} \in d_{\delta}^{p}\right)$, then $p \Vdash_{\mathbb{Q}_{\lambda}^{1}}$ " $A \in \underset{\sim}{D}$ ". Plainly, the family $\left\{A \subseteq \lambda: p \Vdash_{\mathbb{Q}_{\lambda}^{1}}\right.$ " $A \in \underset{\sim}{D}$ " $\}$ is a uniform filter on $\lambda$, and, of course, for a generic filter $G \subseteq \mathbb{Q}_{\lambda}^{1}$ over $\mathbf{V}$,

$$
\stackrel{\sim}{D}^{G}=\bigcup\left\{\left\{A \subseteq \lambda: p \Vdash_{\mathbb{Q}_{\lambda}^{1}} \text { “A } A \in \underset{\sim}{D} "\right\}: p \in G\right\} .
$$

Definition 2.5. (1) We define a forcing notion $\mathbb{Q}_{\lambda}^{0}$ as follows. A condition in $\mathbb{Q}_{\lambda}^{0}$ is a tuple $p=\left(C^{p},\left\langle Z_{\delta}^{p}: \delta \in C^{p}\right\rangle,\left\langle d_{\delta}^{p}: \delta \in C^{p}\right\rangle\right)$ such that $\left(0, C^{p},\left\langle Z_{\delta}^{p}: \delta \in C^{p}\right\rangle,\left\langle d_{\delta}^{p}: \delta \in C^{p}\right\rangle\right) \in \mathbb{Q}_{\lambda}^{1}$. The order $\leq_{\mathbb{Q}_{\lambda}^{0}}=\leq$ of $\mathbb{Q}_{\lambda}^{0}$ is inherited from $\mathbb{Q}_{\lambda}^{1}$ in a natural way.

(2) We define a relation $\leq_{\mathbb{Q}_{\lambda}^{0}}^{*}=\leq^{*}$ on $\mathbb{Q}_{\lambda}^{0}$ as follows: $p \leq^{*} q$ if and only if for some $\alpha<\lambda$ we have

$$
\begin{aligned}
\left(C^{p} \backslash \alpha,\left\langle Z_{\delta}^{p}\right.\right. & \left.\left.: \delta \in C^{p} \backslash \alpha\right\rangle,\left\langle d_{\delta}^{p}: \delta \in C^{p} \backslash \alpha\right\rangle\right) \\
& \leq \mathbb{Q}_{\lambda}^{0}\left(C^{q} \backslash \alpha,\left\langle Z_{\delta}^{q}: \delta \in C^{q} \backslash \alpha\right\rangle,\left\langle d_{\delta}^{q}: \delta \in C^{q} \backslash \alpha\right\rangle\right) .
\end{aligned}
$$


(3) For a condition $q \in \mathbb{Q}_{\lambda}^{0}$ we let

$$
\operatorname{fil}(q):=\left\{A \subseteq \lambda:(\exists \varepsilon<\lambda)\left(\forall \delta \in C^{q} \backslash \varepsilon\right)\left(A \cap Z_{\delta}^{q} \in d_{\delta}^{q}\right)\right\},
$$

and for a set $G^{*} \subseteq \mathbb{Q}_{\lambda}^{0}$ we let $\operatorname{fil}\left(G^{*}\right):=\bigcup\left\{\operatorname{fil}(p): p \in G^{*}\right\}$. We also define a binary relation $\leq^{0}$ on $\mathbb{Q}_{\lambda}^{0}$ by

$$
p \leq^{0} q \text { if and only if } \operatorname{fil}(p) \subseteq \operatorname{fil}(q) .
$$

(4) We say that an ultrafilter $D$ on $\lambda$ is reasonable if it is weakly reasonable (see 1.4(1)) and there is a directed (with respect to $\leq^{0}$ ) set $G^{*} \subseteq \mathbb{Q}_{\lambda}^{0}$ such that $D=\operatorname{fil}\left(G^{*}\right)$. The family $G^{*}$ may be called a generating system for $D$.

(5) An ultrafilter $D$ on $\lambda$ is said to be very reasonable if it is weakly reasonable and there is a $\left(<\lambda^{+}\right)$-directed (with respect to $\leq^{0}$ ) set $G^{*} \subseteq \mathbb{Q}_{\lambda}^{0}$ such that $D=\operatorname{fil}\left(G^{*}\right)$.

REMARK 2.6. Note that $\|\operatorname{fil}(p)\|=2^{\lambda}$ whenever $p \in \mathbb{Q}_{\lambda}^{0}$ is such that all ultrafilters $d_{\alpha}^{p}$ (for $\alpha \in C^{p}$ ) are non-principal. Thus even if $D=\operatorname{fil}\left(G^{*}\right)$ for some small generating system $G^{*} \subseteq \mathbb{Q}_{\lambda}^{0}$, the minimal number of generators for $D$ as a filter may be $2^{\lambda}$.

OBservation 2.7.

(1) If $p \leq_{\mathbb{Q}_{\lambda}^{0}}^{*} q$, then $\operatorname{fil}(p) \subseteq \operatorname{fil}(q)\left(\right.$ so $\left.p \leq^{0} q\right)$.

(2) If a set $G^{*} \subseteq \mathbb{Q}_{\lambda}^{0}$ is directed with respect to $\leq^{0}$, then $\operatorname{fil}\left(G^{*}\right)$ is a filter of subsets of $\lambda$ containing all co-bounded subsets of $\lambda$.

Definition 2.8. Suppose that

(a) $X$ is a non-empty set and $e$ is an ultrafilter on $X$,

(b) $d_{x}$ is an ultrafilter on a set $Z_{x}($ for $x \in X)$.

We let

$$
\bigoplus_{x \in X}^{e} d_{x}=\left\{A \subseteq \bigcup_{x \in X} Z_{x}:\left\{x \in X: Z_{x} \cap A \in d_{x}\right\} \in e\right\}
$$

(Clearly, $\bigoplus_{x \in X}^{e} d_{x}$ is an ultrafilter on $\bigcup_{x \in X} Z_{x}$.)

Proposition 2.9. Let $p, q \in \mathbb{Q}_{\lambda}^{0}$. Then the following are equivalent:

(a) $p \leq^{0} q$,

(b) there is $\varepsilon<\lambda$ such that

$$
\left(\forall \alpha \in C^{q} \backslash \varepsilon\right)\left(\forall A \in d_{\alpha}^{q}\right)\left(\exists \beta \in C^{p}\right)\left(A \cap Z_{\beta}^{p} \in d_{\beta}^{p}\right),
$$

(c) there is $\varepsilon<\lambda$ such that if $\alpha \in C^{q} \backslash \varepsilon$ and $\beta_{0}=\sup \left(C^{p} \cap(\alpha+1)\right)$, $\beta_{1}=\min \left(C^{p} \backslash \min \left(C^{q} \backslash(\alpha+1)\right)\right)$, then there is an ultrafilter $e$ on $\left[\beta_{0}, \beta_{1}\right) \cap C^{p}$ such that

$$
d_{\alpha}^{q}=\left\{A \cap Z_{\alpha}^{q}: A \in \bigoplus^{e}\left\{d_{\beta}^{p}: \beta \in\left[\beta_{0}, \beta_{1}\right) \cap C^{p}\right\}\right\} .
$$


Proof. (a) $\Rightarrow(\mathrm{b})$. Assume towards a contradiction that $p \leq 0$, but (b) fails. Then we may pick a sequence $\left\langle\alpha_{\xi}, A_{\xi}: \xi<\lambda\right\rangle$ such that for each $\xi<\lambda$,

(i) $\alpha_{\xi} \in C^{q}, A_{\xi} \in d_{\alpha_{\xi}}^{q}$,

(ii) if $\xi<\zeta<\lambda, \beta \in C^{p} \cap \min \left(C^{q} \backslash\left(\alpha_{\xi}+1\right)\right)$, then $\min \left(C^{p} \backslash(\beta+1)\right)<\alpha_{\zeta}$,

(iii) $\left(\forall \beta \in C^{p}\right)\left(A_{\xi} \cap Z_{\beta}^{p} \notin d_{\beta}^{p}\right)$.

It follows from (ii) that for every $\beta \in C^{p}$ there is at most one $\xi<\lambda$ such that $Z_{\beta}^{p} \cap Z_{\alpha_{\xi}}^{q} \neq \emptyset$. Also if $\beta \in C^{p}$ and $Z_{\beta}^{p} \cap Z_{\alpha_{\xi}}^{q} \in d_{\beta}^{p}$, then $\left(Z_{\alpha_{\xi}}^{q} \backslash A_{\xi}\right) \cap Z_{\beta}^{p} \in d_{\beta}^{p}$.

Put $A=\bigcup_{\xi<\lambda} A_{\xi}$. By what we have said above, for all $\beta \in C^{p}$ we have $(\lambda \backslash A) \cap Z_{\beta}^{p} \in d_{\beta}^{p}$, and hence $\lambda \backslash A \in \operatorname{fil}(p) \subseteq \operatorname{fil}(q)$. This contradicts (i).

(b) $\Rightarrow$ (c). Assume that (b) holds true as witnessed by $\varepsilon<\lambda$. Let $\alpha \in$ $C^{q} \backslash \varepsilon, \alpha^{\prime}=\min \left(C^{q} \backslash(\alpha+1)\right), \beta_{0}=\sup \left(C^{p} \cap(\alpha+1)\right)$ and $\beta_{1}=\min \left(C^{p} \backslash \alpha^{\prime}\right)$. For $A \in d_{\alpha}^{q}$ put

$$
w(A)=\left\{\beta \in\left[\beta_{0}, \beta_{1}\right) \cap C^{p}: A \cap Z_{\beta}^{p} \in d_{\beta}^{p}\right\} .
$$

It follows from (b) that $w(A) \neq \emptyset$. Plainly $w\left(A \cap A^{\prime}\right)=w(A) \cap w\left(A^{\prime}\right)$ for $A, A^{\prime} \in d_{\alpha}^{q}$, so we may pick an ultrafilter $e$ on $\left[\beta_{0}, \beta_{1}\right) \cap C^{p}$ such that $\left\{w(A): A \in d_{\alpha}^{q}\right\} \subseteq e$. Now it should be clear that

$$
d_{\alpha}^{q} \subseteq\left\{B \cap Z_{\alpha}^{q}: B \in \bigoplus^{e}\left\{d_{\beta}^{p}: \beta \in\left[\beta_{0}, \beta_{1}\right) \cap C^{p}\right\}\right\}
$$

and (since clearly $Z_{\alpha}^{q} \in \bigoplus^{e}\left\{d_{\beta}^{p}: \beta \in\left[\beta_{0}, \beta_{1}\right) \cap C^{p}\right\}$ ) the set on the right-hand side is a proper filter on $Z_{\alpha}^{q}$. Consequently, the two sets are equal.

(c) $\Rightarrow($ a). Assume that (c) holds true as witnessed by $\varepsilon<\lambda$, and suppose that $A \in \operatorname{fil}(p)$. Pick $\varepsilon^{\prime}<\lambda$ such that $\varepsilon<\varepsilon^{\prime}$ and

$$
\left(\forall \beta \in C^{p} \backslash \varepsilon^{\prime}\right)\left(A \cap Z_{\beta}^{p} \in d_{\beta}^{p}\right) .
$$

Suppose $\alpha \in C^{q} \backslash\left(\min \left(C^{p} \backslash \varepsilon^{\prime}\right)+1\right)$ and let $\beta_{0}=\sup \left(C^{p} \cap(\alpha+1), \beta_{1}=\right.$ $\min \left(C^{p} \backslash \min \left(C^{q} \backslash(\alpha+1)\right)\right)$. Let $e$ be an ultrafilter on $\left[\beta_{0}, \beta_{1}\right) \cap C^{p}$ such that

$$
d_{\alpha}^{q}=\left\{B \cap Z_{\alpha}^{q}: B \in \bigoplus^{e}\left\{d_{\beta}^{p}: \beta \in\left[\beta_{0}, \beta_{1}\right) \cap C^{p}\right\}\right\} .
$$

Note that $\beta_{0} \geq \varepsilon^{\prime}$ and hence $A \cap Z_{\beta}^{p} \in d_{\beta}^{p}$ for all $\beta \in\left[\beta_{0}, \beta_{1}\right) \cap C^{p}$. Consequently,

$$
A \cap\left[\beta_{0}, \beta_{1}\right) \in \bigoplus^{e}\left\{d_{\beta}^{p}: \beta \in\left[\beta_{0}, \beta_{1}\right) \cap C^{p}\right\}
$$

and therefore also

$$
A \cap Z_{\alpha}^{q}=\left(A \cap\left[\beta_{0}, \beta_{1}\right)\right) \cap Z_{\alpha}^{q} \in d_{\alpha}^{q} .
$$

Now we easily conclude that $A \in \operatorname{fil}(q)$.

Definition 2.10. Let $p \in \mathbb{Q}_{\lambda}^{0}$. Suppose that $X \in\left[C^{p}\right]^{\lambda}$ and $C \subseteq C^{p}$ is a club of $\lambda$ such that

$$
\text { if } \alpha<\beta \text { are successive elements of } C \text {, then }|[\alpha, \beta) \cap X|=1 \text {. }
$$


(In this situation we say that $p$ is restrictable to $\langle X, C\rangle$.) We define the restriction of $p$ to $\langle X, C\rangle$ as an element $q=p\left\lceil\langle X, C\rangle \in \mathbb{Q}_{\lambda}^{0}\right.$ such that $C^{q}=C$, and if $\alpha<\beta$ are successive elements of $C, x \in[\alpha, \beta) \cap X$, then $Z_{\alpha}^{q}=[\alpha, \beta)$ and $d_{\alpha}^{q}=\left\{A \subseteq Z_{\alpha}^{q}: A \cap Z_{x}^{p} \in d_{x}^{p}\right\}$.

Proposition 2.11.

(1) Assume that $G^{*} \subseteq \mathbb{Q}_{\lambda}^{0}$ is $\leq^{0}$-directed and $\leq^{0}$-downward closed, $p \in$ $G^{*}, X \in\left[C^{p}\right]^{\lambda}$ and $C \subseteq C^{p}$ is a club of $\lambda$ such that $p$ is restrictable to $\langle X, C\rangle$. If $\bigcup_{x \in X} Z_{x}^{p} \in \operatorname{fil}\left(G^{*}\right)$, then $p\left\lceil\langle X, C\rangle \in G^{*}\right.$.

(2) If $G^{*} \subseteq \mathbb{Q}_{\lambda}^{0}$ is $\leq^{0}$-directed and $\left\|G^{*}\right\| \leq \lambda$, then $G^{*}$ has $a \leq^{0}$-upper bound. (Hence, in particular, $\operatorname{fil}\left(G^{*}\right)$ is not an ultrafilter.)

Proof. (1) Suppose that $G^{*}, p, X, C$ are as in the assumptions and $\bigcup_{x \in X} Z_{x}^{p} \in \operatorname{fil}\left(G^{*}\right)$. Since $G^{*}$ is $\leq^{0}$-directed (and $p \in G^{*}$ ) we may pick $r \in G^{*}$ such that $p \leq^{0} r$ and $\bigcup_{x \in X} Z_{x}^{p} \in \operatorname{fil}(r)$. We are going to show that $q:=p \nmid\langle X, C\rangle \leq{ }^{0} r$ (which will imply that $q \in G^{*}$ as $G^{*}$ is downward closed).

Since $\bigcup_{x \in X} Z_{x}^{p} \in \operatorname{fil}(r)$, there is $\varepsilon<\lambda$ such that

$$
\left(\forall \alpha \in C^{r} \backslash \varepsilon\right)\left(\bigcup_{x \in X} Z_{x}^{p} \cap Z_{\alpha}^{r} \in d_{\alpha}^{r}\right)
$$

and

$$
\left(\forall \alpha \in C^{r} \backslash \varepsilon\right)\left(\forall A \in d_{\alpha}^{r}\right)\left(\exists \beta \in C^{p}\right)\left(A \cap Z_{\beta}^{p} \in d_{\beta}^{p}\right)
$$

(remember 2.9(b)). Now suppose that $\alpha \in C^{r} \backslash \varepsilon$ and $A \in d_{\alpha}^{r}$. Then $\bigcup_{x \in X} Z_{x}^{p}$ $\cap A \in d_{\alpha}^{r}$ so there is $\beta \in C^{p}$ such that $\bigcup_{x \in X} Z_{x}^{p} \cap A \cap Z_{\beta}^{p} \in d_{\beta}^{p}$. In particular, $\bigcup_{x \in X} Z_{x}^{p} \cap Z_{\beta}^{p} \cap A \neq \emptyset$, so necessarily $\beta \in X$. Let $\beta_{0}<\beta_{1}$ be the successive elements of $C$ such that $\beta_{0} \leq \beta<\beta_{1}$. Since

$$
Z_{\beta}^{p} \cap A=\bigcup_{x \in X} Z_{x}^{p} \cap Z_{\beta}^{p} \cap A \in d_{\beta}^{p},
$$

we also have $A \cap Z_{\beta_{0}}^{q} \in d_{\beta_{0}}^{q}$. Thus we have shown that

if $\alpha \in C^{r} \backslash \varepsilon$ and $A \in d_{\alpha}^{r}$, then there is $\beta_{0} \in C^{q}$ such that $A \cap Z_{\beta_{0}}^{q} \in d_{\beta_{0}}^{q}$.

Consequently, $q \leq^{0} r$ (remember 2.9).

(2) Let $\left\langle p_{\xi}: \xi<\lambda\right\rangle$ list (with possible repetitions) all members of $G^{*}$. For $\xi<\lambda$ let $C_{\xi}=\left\{\delta<\lambda: \delta=\sup \left(\delta \cap C^{p_{\xi}}\right)\right\}$ (it is a club of $\lambda$ ), and for $\xi, \zeta<\lambda$ let $\varepsilon(\{\xi, \zeta\})<\lambda$ be such that if $p_{\xi} \leq{ }^{0} p_{\zeta}$, then

$$
\left(\forall \alpha \in C^{p_{\zeta}} \backslash \varepsilon(\{\xi, \zeta\})\right)\left(\forall A \in d_{\alpha}^{p_{\zeta}}\right)\left(\exists \beta \in C^{p_{\xi}}\right)\left(A \cap Z_{\beta}^{p_{\xi}} \in d_{\beta}^{p_{\xi}}\right)
$$

(remember 2.9). Let

$$
C^{*}=\left\{\delta<\lambda: \delta \text { is limit and }\left\{p_{\xi}: \xi<\delta\right\} \text { is } \leq^{0} \text {-directed }\right\}
$$

(again, it is a club of $\lambda$ ). Finally, let

$$
C=\left\{\delta \in C^{*} \cap \triangle_{\xi<\lambda} C_{\xi}:(\forall \xi, \zeta<\delta)(\varepsilon(\{\xi, \zeta\})<\delta)\right\} .
$$


Plainly, $C$ is a club of $\lambda$. Now, suppose that $\delta<\gamma$ are two successive members of $C$. Put $Z_{\delta}=[\delta, \gamma)$ and let

$$
I_{\delta}=\left\{A \subseteq Z_{\delta}:(\exists \xi<\delta)\left(\forall \alpha \in C^{p_{\xi}} \backslash \delta\right)\left(A \cap Z_{\alpha}^{p_{\xi}} \notin d_{\alpha}^{p_{\xi}}\right)\right\} .
$$

It easily follows from the definition of $C$ that $I_{\delta}$ is a proper ideal on $Z_{\delta}$, so we may pick an ultrafilter $d_{\delta}$ on $Z_{\delta}$ disjoint from $I_{\delta}$. Let $q=\left(C,\left\langle Z_{\delta}: \delta \in C\right\rangle\right.$, $\left\langle d_{\delta}: \delta \in C\right\rangle$ ). Clearly $q \in \mathbb{Q}_{\lambda}^{0}$ and we will argue that $q$ is a $\leq^{0}$-upper bound to $G^{*}$. So let $\xi<\lambda$. Suppose that $\delta \in C \backslash(\xi+1)$ and $A \in d_{\delta}$. Then $A \notin I_{\delta}$, so there is $\alpha \in C^{p_{\xi}} \backslash \delta$ such that $A \cap Z_{\alpha}^{p_{\xi}} \in d_{\alpha}^{p_{\xi}}$. Now we may use 2.9 to conclude that $p_{\xi} \leq^{0} q$.

Proposition 2.12. If $2^{\lambda}=\lambda^{+}$, then there is a $\leq_{\mathbb{Q}_{\lambda}^{0}}^{*}$-increasing sequence $\bar{p}=\left\langle p_{\varepsilon}: \varepsilon\left\langle\lambda^{+}\right\rangle \subseteq \mathbb{Q}_{\lambda}^{0}\right.$ such that

$$
\operatorname{fil}(\bar{p}):=\bigcup\left\{\operatorname{fil}\left(p_{\varepsilon}\right): \varepsilon<\lambda^{+}\right\}
$$

is a uniform ultrafilter on $\lambda$.

Proof. Straightforward induction using 2.3(4) and the proof of 2.11(2).

For basic information on the ideal of meager subsets of ${ }^{\lambda} \lambda$ and its covering number we refer the reader e.g. to Matet, Rosłanowski and Shelah [6, §4]. Here we state only the definitions we will need.

DEFINITION 2.13.

(1) The space ${ }^{\lambda} \lambda$ is endowed with the topology obtained by taking as basic open sets $\emptyset$ and $O_{s}$ for $s \in{ }^{\lambda>} \lambda$, where $O_{s}=\left\{f \in{ }^{\lambda} \lambda: s \subseteq f\right\}$.

(2) The $(<\lambda)$-complete ideal of subsets of ${ }^{\lambda} \lambda$ generated by nowhere dense subsets of ${ }^{\lambda} \lambda$ is denoted by $\mathbf{M}_{\lambda, \lambda}^{\lambda}$.

(3) $\operatorname{cov}\left(\mathbf{M}_{\lambda, \lambda}^{\lambda}\right)$ is the minimal size of a family $\mathcal{A} \subseteq \mathbf{M}_{\lambda, \lambda}^{\lambda}$ such that $\cup \mathcal{A}={ }^{\lambda} \lambda$.

Theorem 2.14. Assume that $\lambda^{<\lambda}=\lambda$ and $\operatorname{cov}\left(\mathbf{M}_{\lambda, \lambda}^{\lambda}\right)=2^{\lambda}$. Then there exists a very reasonable ultrafilter on $\lambda$.

Proof. Fix a model $N \prec \mathcal{H}(\chi)$ (for some large regular cardinal $\chi$ ) such that $\|N\|=\lambda$ and ${ }^{\lambda>} N \subseteq N$.

For $p \in \mathbb{Q}_{\lambda}^{0}$ let $\left\langle\delta_{\alpha}^{p}: \alpha<\lambda\right\rangle$ be the increasing enumeration of $C^{p}$ and let $\eta^{p}$ be the sequence of length $\lambda$ such that

Next let

$$
(\forall \alpha<\lambda)\left(\eta^{p}(\alpha)=\left\langle Z_{\delta_{\alpha}^{p}}^{p}, d_{\delta_{\alpha}^{p}}^{p}\right\rangle\right)
$$

$$
\mathcal{T}_{\alpha}=\left\{\eta^{p} \mid \alpha: p \in \mathbb{Q}_{\lambda}^{0}\right\} \cap N \quad(\text { for } \alpha<\lambda) \quad \text { and } \quad \mathcal{T}=\bigcup_{\alpha<\lambda} \mathcal{T}_{\alpha} .
$$

Clearly $\mathcal{T}$ is a tree isomorphic to ${ }^{\lambda>} \lambda$ by an isomorphism preserving the levels (i.e., mapping $\mathcal{T}_{\alpha}$ onto ${ }^{\alpha} \lambda$ ). Also, every $\lambda$-branch $\eta \in \lim (\mathcal{T})$ determines a condition $p \in \mathbb{Q}_{\lambda}^{0}$ such that $\eta=\eta^{p}$. Let $Q^{*}=\left\{p \in \mathbb{Q}_{\lambda}^{0}: \eta^{p} \in \lim (\mathcal{T})\right\}$. 
A family $G^{*} \subseteq Q^{*}$ is linked if it is $(<\omega)$-linked with respect to the partial order $\leq^{0}$-restricted to $Q^{*}$, that is, if every finite subset of $G^{*}$ has a $\leq^{0}$-upper bound in $Q^{*}$ (but the bound does not have to be in $G^{*}$ ). Note that if $p_{0}, \ldots, p_{n} \in Q^{*}$ have a $\leq^{0}$-upper bound in $\mathbb{Q}_{\lambda}^{0}$, then they have a $\leq^{0}$-upper bound in $Q^{*}$ as well. For $p_{0}, \ldots, p_{n} \in Q^{*}, \delta<\delta^{\prime}<\lambda$ and an ultrafilter $d$ on $\left[\delta, \delta^{\prime}\right)$ let $(\oplus)^{p_{0}, \ldots, p_{n}}\left(\delta, \delta^{\prime}, d\right)$ mean

$(\oplus)^{p_{0}, \ldots, p_{n}}$ (a) $\delta, \delta^{\prime} \in C^{p_{0}} \cap \ldots \cap C^{p_{n}}$, and

(b) if $B \in d, i \leq n$, then there is $\xi \in\left[\delta, \delta^{\prime}\right) \cap C^{p_{i}}$ such that $B \cap Z_{\xi}^{p_{i}} \in d_{\xi}^{p_{i}}$.

Claim 2.14.1. If $G^{*} \subseteq Q^{*}$ is a linked family, $\left\|G^{*}\right\|<\operatorname{cov}\left(\mathbf{M}_{\lambda, \lambda}^{\lambda}\right)$, and $A \subseteq \lambda$, then there is $p \in Q^{*}$ such that

(a) $G^{*} \cup\{p\}$ is linked,

(b) either $A \in \operatorname{fil}(p)$ or $\lambda \backslash A \in \operatorname{fil}(p)$.

Proof of the Claim. We will consider two cases.

CASE 1: For every $p_{0}, \ldots, p_{n} \in G^{*}, n<\omega$, there is $p \in Q^{*}$ such that $A \in \operatorname{fil}(p)$ and $p_{0} \leq^{0} p, \ldots, p_{n} \leq^{0} p$. Note that the assumption of the present case is equivalent to

$(\otimes)$ for every $p_{0}, \ldots, p_{n} \in G^{*}, n<\omega$, and $\alpha<\lambda$ there are $\delta<\delta^{\prime}<\lambda$ and an ultrafilter $d \in N$ on $\left[\delta, \delta^{\prime}\right)$ such that $(\oplus)^{p_{0}, \ldots, p_{n}}\left(\delta, \delta^{\prime}, d\right)$ holds true and $\alpha<\delta$ and $A \cap\left[\delta, \delta^{\prime}\right) \in d$.

We let

$$
\mathcal{T}_{A}=\{\eta \in \mathcal{T}:(\forall \alpha<\operatorname{lh}(\eta))(\forall Z, d)(\eta(\alpha)=\langle Z, d\rangle \Rightarrow A \cap Z \in d)\} .
$$

Clearly, $\mathcal{T}_{A}$ is a $\lambda$-branching subtree of $\mathcal{T}$ and $\mathcal{T}_{A}$ is isomorphic to ${ }^{\lambda>\lambda}$. Now, for $p_{0}, \ldots, p_{n} \in G^{*}, n<\omega$, and $\alpha<\lambda$ let $I_{\alpha}^{A}\left(p_{0}, \ldots, p_{n}\right)$ be the set $\left\{\eta \in \lim \left(\mathcal{T}_{A}\right):(\exists \beta>\alpha)\left(\exists \delta, \delta^{\prime}, d\right)\left((\oplus)^{p_{0}, \ldots, p_{n}}\left(\delta, \delta^{\prime}, d\right) \& \eta(\beta)=\left\langle\left[\delta, \delta^{\prime}\right), d\right\rangle\right)\right\}$.

It should be clear that $I_{\alpha}^{A}\left(p_{0}, \ldots, p_{n}\right)$ is an open dense subset of $\lim \left(\mathcal{T}_{A}\right)$ (remember $(\otimes))$. Therefore (as $\left.\left\|G^{*}\right\|<\operatorname{cov}\left(\mathbf{M}_{\lambda, \lambda}^{\lambda}\right)\right)$ we know that

$$
\bigcap\left\{I_{\alpha}^{A}\left(p_{0}, \ldots, p_{n}\right): n<\omega \& p_{0}, \ldots, p_{n} \in G^{*} \& \alpha<\lambda\right\} \neq \emptyset
$$

and we may choose $\eta$ from the set on the left-hand side above. Let $p \in Q^{*}$ be such that $\eta=\eta^{p}$. Since $\eta \in \lim \left(\mathcal{T}_{A}\right)$ we know that $A \in \operatorname{fil}(p)$. Also, for every $p_{0}, \ldots, p_{n} \in G^{*}$ we have $\eta \in \bigcap_{\alpha<\lambda} I_{\alpha}^{A}\left(p_{0}, \ldots, p_{n}\right)$ and hence

$$
\|\left\{\delta \in C^{p}: \text { if } \delta^{\prime}=\min \left(C^{p} \backslash(\delta+1)\right) \text { then }(\oplus)^{p_{0}, \ldots, p_{n}}\left(\delta, \delta^{\prime}, d_{\delta}^{p}\right)\right\} \|=\lambda .
$$

So one may easily construct $p^{*} \in Q^{*}$ which is $\leq^{0}$-stronger than $p, p_{0}, \ldots, p_{n}$ (remember 2.9). Thus we have justified that $G^{*} \cup\{p\}$ is linked.

CASE 2: There are $p_{0}, \ldots, p_{n} \in G^{*}, n<\omega$, such that if $p \in Q^{*}$ is $\leq^{0}$-stronger than $p_{0}, \ldots, p_{n}$, then $A \notin \operatorname{fil}(p)$. 
It follows from the proof of 2.3(4) that then

for every $q_{0}, \ldots, q_{m} \in G^{*}, m<\omega$, there is $q \in Q^{*}$ such that

$\lambda \backslash A \in \operatorname{fil}(q)$ and $q_{0} \leq^{0} q, \ldots, q_{m} \leq^{0} q$

(remember $G^{*}$ is linked and that bounded subsets of $\lambda$ are in $N$ ). Thus we may repeat the arguments of Case 1 for $\lambda \backslash A$ and we find $p \in Q^{*}$ such that $G^{*} \cup\{p\}$ is linked and $\lambda \backslash A \in \operatorname{fil}(p)$.

Claim 2.14.2. If $G^{*} \subseteq Q^{*}$ is linked, $\left\|G^{*}\right\|<\operatorname{cov}\left(\mathbf{M}_{\lambda, \lambda}^{\lambda}\right)$ and $p_{0}, p_{1} \in G^{*}$, then there is $p \in Q^{*}$ such that

(a) $G^{*} \cup\{p\}$ is linked,

(b) $p_{0} \leq^{0} p$ and $p_{1} \leq^{0} p$.

Proof of the Claim. Let $p_{0}, p_{1} \in G^{*}$. Note that

$(\odot)$ for every $p_{2}, \ldots, p_{n} \in G^{*}, 2 \leq n<\omega$, and $\alpha<\lambda$ there are $\delta<\delta^{\prime}<\lambda$ and an ultrafilter $d \in N$ on $\left[\delta, \delta^{\prime}\right)$ such that $(\oplus)^{p_{0}, p_{1}, p_{2}, \ldots, p_{n}}\left(\delta, \delta^{\prime}, d\right)$ holds true and $\alpha<\delta$.

We let $\mathcal{T}^{p_{0}, p_{1}}$ be the set

$$
\left\{\eta \in \mathcal{T}:(\forall \alpha<\operatorname{lh}(\eta))\left(\forall \delta, \delta^{\prime}, d\right)\left(\eta(\alpha)=\left\langle\left[\delta, \delta^{\prime}\right), d\right\rangle \Rightarrow(\oplus)^{p_{0}, p_{1}}\left(\delta, \delta^{\prime}, d\right)\right)\right\}
$$

and we note that $\mathcal{T}^{p_{0}, p_{1}}$ is a $\lambda$-branching subtree of $\mathcal{T}$ isomorphic to ${ }^{\lambda>} \lambda$. For $p_{2}, \ldots, p_{n} \in G^{*}, 2 \leq n<\omega$, and $\alpha<\lambda$ we let $I_{\alpha}^{p_{0}, p_{1}}\left(p_{2}, \ldots, p_{n}\right)$ be the set

$$
\begin{aligned}
\left\{\eta \in \lim \left(\mathcal{T}^{p_{0}, p_{1}}\right):(\exists \beta>\alpha)\left(\exists \delta, \delta^{\prime}, d\right)\left((\oplus)^{p_{2}, \ldots, p_{n}}\left(\delta, \delta^{\prime}, d\right) \&\right.\right. \\
\left.\left.\eta(\beta)=\left\langle\left[\delta, \delta^{\prime}\right), d\right\rangle\right)\right\} .
\end{aligned}
$$

Then $I_{\alpha}^{p_{0}, p_{1}}\left(p_{2}, \ldots, p_{n}\right)$ is an open dense subset of $\lim \left(\mathcal{T}^{p_{0}, p_{1}}\right)$ (remember $(\odot))$. Since $\left\|G^{*}\right\|<\operatorname{cov}\left(\mathbf{M}_{\lambda, \lambda}^{\lambda}\right)$, we may choose $p \in Q^{*}$ such that

$$
\eta^{p} \in \bigcap\left\{I_{\alpha}^{p_{0}, p_{1}}\left(p_{2}, \ldots, p_{n}\right): 2 \leq n<\omega \& p_{2}, \ldots, p_{n} \in G^{*} \& \alpha<\lambda\right\} \neq \emptyset .
$$

As in the proof of 2.14.1 we argue that $G^{*} \cup\{p\}$ is linked. Since $\eta^{p} \in$ $\lim \left(\mathcal{T}^{p_{0}, p_{1}}\right)$ we easily see that $p$ is $\leq^{0}$-stronger than both $p_{0}$ and $p_{1}$.

Claim 2.14.3. If $G^{*} \subseteq Q^{*}$ is a linked family, $\left\|G^{*}\right\|<\operatorname{cov}\left(\mathbf{M}_{\lambda, \lambda}^{\lambda}\right), \xi \leq \lambda$ is a limit ordinal and a sequence $\left\langle p_{\zeta}: \zeta<\xi\right\rangle \subseteq G^{*}$ is $\leq^{0}$-increasing, then there is $p \in Q^{*}$ such that

(a) $G^{*} \cup\{p\}$ is linked,

(b) $(\forall \zeta<\xi)\left(p_{\zeta} \leq^{0} p\right)$.

Proof of the Claim. First let us consider the case when $\xi<\lambda$. Suppose that a sequence $\bar{p}=\left\langle p_{\zeta}: \zeta<\xi\right\rangle \subseteq G^{*}$ is $\leq^{0}$-increasing and let

$$
\begin{aligned}
\mathcal{T}_{\bar{p}}=\left\{\eta \in \mathcal{T}:(\forall \alpha<\operatorname{lh}(\eta))\left(\forall \delta, \delta^{\prime}, d\right)(\eta(\alpha)\right. & =\left\langle\left[\delta, \delta^{\prime}\right), d\right\rangle \\
& \left.\left.\Rightarrow(\forall \zeta<\xi)(\oplus)^{p_{\zeta}}\left(\delta, \delta^{\prime}, d\right)\right)\right\}
\end{aligned}
$$


By arguments similar to that of $2.3(3)$ we verify that $\mathcal{T}_{\bar{p}}$ is a $\lambda$-branching subtree of $\mathcal{T}$ and it is isomorphic to ${ }^{\lambda>} \lambda$. As in the previous claims, for $p_{0}^{\prime}, \ldots, p_{n}^{\prime} \in G^{*}, n<\omega$, and $\alpha<\lambda$ we let $I_{\alpha}^{\bar{p}}\left(p_{0}^{\prime}, \ldots, p_{n}^{\prime}\right)$ be the set

$$
\left\{\eta \in \lim \left(\mathcal{T}_{\bar{p}}\right):(\exists \beta>\alpha)\left(\exists \delta, \delta^{\prime}, d\right)\left((\oplus)^{p_{0}^{\prime}, \ldots, p_{n}^{\prime}}\left(\delta, \delta^{\prime}, d\right) \& \eta(\beta)=\left\langle\left[\delta, \delta^{\prime}\right), d\right\rangle\right)\right\} .
$$

Then each $I_{\alpha}^{\bar{p}}\left(p_{0}^{\prime}, \ldots, p_{n}^{\prime}\right)$ is an open dense subset of $\lim \left(\mathcal{T}_{\bar{p}}\right)$. [Why? Let $\eta \in \mathcal{T}_{\bar{p}}$. We may assume that for each $\varepsilon<\zeta<\xi$ and $\beta \in C^{p_{\zeta}} \backslash \operatorname{lh}(\eta)$ and $A \in d_{\beta}^{p_{\zeta}}$ there is $\gamma \in C^{p_{\varepsilon}}$ such that $A \cap Z_{\gamma}^{p_{\varepsilon}} \in d_{\gamma}^{p_{\varepsilon}}$. We also may demand that $\delta_{0}:=\sup \left(\delta^{\prime}<\lambda:(\exists \alpha<\operatorname{lh}(\eta))(\exists \delta, d)\left(\eta(\alpha)=\left\langle\left[\delta, \delta^{\prime}\right), d\right\rangle\right)\right) \in \bigcap_{\varepsilon<\xi} C^{p_{\varepsilon}} \cap \bigcap_{i \leq n} C^{p_{i}^{\prime}}$.

Choose inductively a sequence $\left\langle\delta_{\zeta}, d_{\zeta}: \zeta<\xi\right\rangle$ so that

(a) $\left\langle\delta_{\zeta}: \zeta<\xi\right\rangle$ is an increasing continuous sequence of ordinals below $\lambda$,

(b) $d_{\zeta} \in N$ is an ultrafilter on $\left[\delta_{\zeta}, \delta_{\zeta+1}\right), \delta_{\zeta+1} \in \bigcap_{\varepsilon<\xi} C^{p_{\varepsilon}}$,

(c) $(\oplus)^{p_{0}^{\prime}, \ldots, p_{n}^{\prime}, p_{\zeta}}\left(\delta_{\zeta}, \delta_{\zeta+1}, d_{\zeta}\right)$ holds true (for each $\zeta<\xi$ ).

Let $\delta_{\xi}=\sup \left(\delta_{\zeta}: \zeta<\xi\right)$ and let $e \in N$ be an ultrafilter on $\xi$ containing all co-bounded subsets of $\xi$. Put $d=\bigoplus_{\zeta<\xi}^{e} d_{\zeta}$ it is an ultrafilter on $\left[\delta_{0}, \delta_{\xi}\right)$, $d \in N$ and $(\oplus)^{p_{0}^{\prime}, \ldots, p_{n}^{\prime}, p_{\zeta}}\left(\delta_{0}, \delta_{\xi}, d\right)$ holds true for each $\zeta<\xi$. Consequently, $\eta \cup\left\{\left(\operatorname{lh}(\eta),\left\langle\left[\delta_{0}, \delta_{\xi}\right), d\right\rangle\right)\right\} \in \mathcal{T}_{\bar{p}}$ and every member of $\lim \left(\mathcal{T}_{\bar{p}}\right)$ extending it belongs to $I_{\alpha}^{\bar{p}}\left(p_{0}^{\prime}, \ldots, p_{n}^{\prime}\right)$.]

Thus we may pick $p \in Q^{*}$ such that

$$
\eta^{p} \in \bigcap\left\{I_{\alpha}^{\bar{p}}\left(p_{0}^{\prime}, \ldots, p_{n}^{\prime}\right): n<\omega \& p_{0}^{\prime}, \ldots, p_{n}^{\prime} \in G^{*} \& \alpha<\lambda\right\} .
$$

Since $\eta^{p} \in \lim \left(\mathcal{T}_{\bar{p}}\right)$ we easily see that $p_{\zeta} \leq^{0} p$ for all $\zeta<\xi$, and as in the proof of 2.14.1 we argue that $G^{*} \cup\{p\}$ is linked.

If $\xi=\lambda$ and $\bar{p}=\left\langle p_{\zeta}: \zeta<\lambda\right\rangle$ is $\leq^{0}$-increasing, then we proceed in a similar manner except that we work in the tree

$$
\begin{aligned}
\mathcal{T}_{\bar{p}}^{*}=\left\{\eta \in \mathcal{T}:(\forall \alpha<\operatorname{lh}(\eta))\left(\forall \delta, \delta^{\prime}, d\right)\left(\eta(\alpha)=\left\langle\left[\delta, \delta^{\prime}\right), d\right\rangle\right.\right. \\
\left.\left.\Rightarrow(\forall \zeta<\alpha)(\oplus)^{p_{\zeta}}\left(\delta, \delta^{\prime}, d\right)\right)\right\}
\end{aligned}
$$

Claim 2.14.4. Assume that $G^{*} \subseteq Q^{*}$ is a linked family, $\left\|G^{*}\right\|<$ $\operatorname{cov}\left(\mathbf{M}_{\lambda, \lambda}^{\lambda}\right), C \subseteq \lambda$ is a club and $\left\langle\delta_{\xi}: \xi<\lambda\right\rangle$ is the increasing enumeration of $C$. Then there is $p \in Q^{*}$ and a club $C^{*}$ of $\lambda$ such that

(a) $G^{*} \cup\{p\}$ is linked,

(b) $\bigcup\left\{\left[\delta_{\xi+1}, \delta_{\zeta}\right): \xi<\zeta\right.$ are successive members of $\left.C^{*}\right\} \in \operatorname{fil}(p)$.

Proof of the Claim. Let

$\mathcal{T}_{C}=\left\{\eta \in \mathcal{T}\right.$ : for each $\alpha<\operatorname{lh}(\eta)$ such that $\alpha=\delta_{\alpha}$ and for every $\alpha^{\prime}, d$,

$$
\left.\left.\eta(\alpha)=\left\langle\left[\alpha, \alpha^{\prime}\right), d\right\rangle \Rightarrow \delta_{\alpha+1}<\alpha^{\prime} \&\left[\delta_{\alpha}, \delta_{\alpha+1}\right) \notin d\right)\right\} .
$$

One easily verifies that $\mathcal{T}_{C}$ is a $\lambda$-branching subtree of $\mathcal{T}$ which is isomorphic to ${ }^{\lambda>\lambda}$. As before, for $p_{0}, \ldots, p_{n} \in G^{*}, n<\omega$, and $\alpha<\lambda$ we let $I_{\alpha}^{C}\left(p_{0}, \ldots, p_{n}\right)$ 
be the set

$$
\left\{\eta \in \lim \left(\mathcal{T}_{C}\right):(\exists \beta>\alpha)\left(\exists \delta, \delta^{\prime}, d\right)\left((\oplus)^{p_{0}, \ldots, p_{n}}\left(\delta, \delta^{\prime}, d\right) \& \eta(\beta)=\left\langle\left[\delta, \delta^{\prime}\right), d\right\rangle\right)\right\} .
$$

Each $I_{\alpha}^{C}\left(p_{0}, \ldots, p_{n}\right)$ is an open dense subset of $\lim \left(\mathcal{T}_{C}\right)$ and hence there is $p \in Q^{*}$ such that

$$
\eta^{p} \in \bigcap\left\{I_{\alpha}^{C}\left(p_{0}, \ldots, p_{n}\right): n<\omega \& p_{0}, \ldots, p_{n} \in G^{*} \& \alpha<\lambda\right\} .
$$

As in the proof of 2.14.1 we argue that $G^{*} \cup\{p\}$ is linked. Put

$$
C^{*}=\left\{\alpha<\lambda: \alpha=\delta_{\alpha} \text { is limit \& }\left(\exists \alpha^{\prime}, d\right)\left(\eta^{p}(\alpha)=\left\langle\left[\alpha, \alpha^{\prime}\right), d\right\rangle\right)\right\}
$$

and note that $C^{*}$ is a club of $\lambda$. Note that if $\alpha \in C^{*}$ and $\eta^{p}(\alpha)=\left\langle\left[\alpha, \alpha^{\prime}\right), d\right\rangle$, then $\delta_{\alpha+1}<\alpha^{\prime}$ and $\left[\delta_{\alpha}, \delta_{\alpha+1}\right) \notin d$. Consequently,

$$
\bigcup\left\{\left[\delta_{\alpha+1}, \delta_{\beta}\right): \alpha<\beta \text { are successive members of } C^{*}\right\} \in \operatorname{fil}(p) .
$$

To prove the theorem we construct inductively a sequence $\left\langle q_{\zeta}: \zeta<2^{\lambda}\right\rangle$ of elements of $Q^{*}$ such that

- for each $\xi<2^{\lambda}$ the family $\left\{q_{\zeta}: \zeta<\xi\right\}$ is linked,

- for each $A \subseteq \lambda$ there is $\zeta<2^{\lambda}$ such that either $A \in \operatorname{fil}\left(q_{\zeta}\right)$ or $\lambda \backslash A \in$ $\operatorname{fil}\left(q_{\zeta}\right)$,

- for each $\zeta<\xi<2^{\lambda}$ there is $\alpha<2^{\lambda}$ such that $q_{\zeta} \leq{ }^{0} q_{\alpha}$ and $q_{\xi} \leq^{0} q_{\alpha}$,

- if $\xi \leq \lambda$ and $\left\langle p_{\zeta}: \zeta<\xi\right\rangle$ is a $\leq^{0}$-increasing sequence of elements of $\left\{q_{\zeta}: \zeta<2^{\lambda}\right\}$, then there is $\alpha<2^{\lambda}$ such that $q_{\alpha}$ is a $\leq^{0}$-upper bound to all $p_{\zeta}$ 's,

- if a sequence $\left\langle\delta_{\xi}: \xi\langle\lambda\rangle \subseteq \lambda\right.$ is increasing continuous, then for some $\zeta<2^{\lambda}$ and a club $C^{*}$ of $\lambda$ we have

$$
\bigcup\left\{\left[\delta_{\xi+1}, \delta_{\xi^{\prime}}\right): \xi<\xi^{\prime} \text { are successive members of } C^{*}\right\} \in \operatorname{fil}\left(q_{\zeta}\right) \text {. }
$$

The construction is a straightforward application of a suitable bookkeeping device and Claims 2.14.1-2.14.4. After it is carried out put $G^{*}=\left\{q_{\zeta}\right.$ : $\left.\zeta<2^{\lambda}\right\}$ and note that $\operatorname{fil}\left(G^{*}\right)$ is a very reasonable ultrafilter on $\lambda$.

Let us finish this section with an observation showing that the assumption $\lambda^{<\lambda}=\lambda$ in Theorem 2.14 is very natural in the given context.

Proposition 2.15. Assume $\theta<\lambda=\operatorname{cf}(\lambda)<2^{\theta}$. Then $\operatorname{cov}\left(\mathbf{M}_{\lambda, \lambda}^{\lambda}\right)=\lambda^{+}$.

Proof. Let $\left\langle\nu_{\xi}: \xi<\lambda^{+}\right\rangle$be a sequence of distinct functions from $\theta$ to 2. Let $\left\langle\delta_{\alpha}: \alpha<\lambda\right\rangle \subseteq \lambda$ be an increasing continuous sequence such that $\delta_{0}=0$, $\delta_{\alpha+1}=\delta_{\alpha}+\theta$ (for $\left.\alpha<\lambda\right)$. Now, for $\xi<\lambda^{+}$we define

$$
F_{\xi}=\left\{\eta \in{ }^{\lambda} \lambda:(\forall \alpha<\lambda)(\exists i<\theta)\left(\eta\left(\delta_{\alpha}+i\right) \neq \nu_{\xi}(i)\right)\right\} .
$$

Plainly, each $F_{\xi}$ is a closed nowhere dense subset of ${ }^{\lambda} \lambda$. We claim that $\bigcup_{\xi<\lambda^{+}} F_{\xi}={ }^{\lambda} \lambda$. To this end suppose that $\eta \in{ }^{\lambda} \lambda$ and consider the restrictions $\eta\left\lceil\left[\delta_{\alpha}, \delta_{\alpha+1}\right)\right.$ for $\alpha<\lambda$. These restrictions determine $\lambda$ functions from $\theta$ 
to 2, so we may find $\xi<\lambda^{+}$such that $\nu_{\xi}$ is distinct from all these functions, i.e., $(\forall \alpha<\lambda)(\exists i<\theta)\left(\eta\left(\delta_{\alpha}+i\right) \neq \nu_{\xi}(i)\right)$. Then $\eta \in F_{\xi}$.

3. fil $\left(G^{*}\right)$ and dominating families. In this section we show that families $G^{*} \subseteq \mathbb{Q}_{\lambda}^{0}$ generating reasonable ultrafilters cannot be too small.

TheOREM 3.1. For $p \in \mathbb{Q}_{\lambda}^{0}$ let $f_{p} \in{ }^{\lambda} \lambda$ be such that

$$
(\forall \alpha<\lambda)\left(f_{p}(\alpha) \in C^{p} \& \operatorname{otp}\left(C^{p} \cap f_{p}(\alpha)\right)=\omega \cdot \alpha+\omega\right) .
$$

(1) Suppose that $G_{0}^{*} \subseteq \mathbb{Q}_{\lambda}^{0}$ is $\left(<\aleph_{1}\right)$-directed (with respect to $\leq^{0}$ ) and $\operatorname{fil}\left(G_{0}^{*}\right)$ is a weakly reasonable ultrafilter. Then $\mathcal{F}_{0}=\left\{f_{p}: p \in G_{0}^{*}\right\}$ is a dominating family in ${ }^{\lambda} \lambda$.

(2) Suppose that $G_{1}^{*} \subseteq \mathbb{Q}_{\lambda}^{0}$ is directed (with respect to $\leq^{0}$ ) and $\operatorname{fil}\left(G_{1}^{*}\right)$ is a weakly reasonable ultrafilter on $\lambda$. Then $\mathcal{F}_{1}=\left\{f_{p}: p \in G_{1}^{*}\right\}$ is a club-dominating family in ${ }^{\lambda} \lambda$.

Proof. (1) First note that if $p, q \in G_{0}^{*}, p \leq^{0} q$, then for some $\varepsilon<\lambda$, if $\alpha<\beta<\gamma$ are successive members of $C^{q} \backslash \varepsilon$, then $(\alpha, \gamma) \cap C^{p} \neq \emptyset$. Thus $p \leq^{0} q$ implies that for all sufficiently large $\alpha<\lambda$ we have $f_{p}(\alpha) \leq f_{q}(\alpha)$. Consequently, the family $\mathcal{F}_{0}$ is $\left(<\aleph_{1}\right.$ )-directed (with respect to $\leq^{*}$ ).

Suppose towards a contradiction that $\mathcal{F}_{0}$ is not a dominating family. Then we may choose an increasing continuous sequence $\bar{\alpha}^{0}=\left\langle\alpha_{\xi}^{0}: \xi\langle\lambda\rangle\right.$ such that

$$
\left(\forall p \in G_{0}^{*}\right)\left(\exists^{\lambda} \varepsilon<\lambda\right)\left(f_{p}\left(\alpha_{\varepsilon}^{0}\right)<\alpha_{\varepsilon+1}^{0}\right) .
$$

Now, by induction on $n<\omega$, choose increasing continuous sequences $\bar{\alpha}^{n}=$ $\left\langle\alpha_{\xi}^{n}: \xi<\lambda\right\rangle$ so that letting $C_{n}=\left\{\alpha_{\xi}^{n}: \xi<\lambda\right\}$ we have

(i) $\bar{\alpha}^{0}$ is the one chosen earlier,

(ii) $C_{n+1} \subseteq\left\{\alpha_{\varepsilon}^{n}: \varepsilon=\alpha_{\varepsilon}^{n}\right.$ is a limit ordinal $\}$,

(iii) $\bigcup\left\{\left[\alpha_{\varepsilon}^{n}, \alpha_{\varepsilon+1}^{n}\right): \varepsilon \in C_{n+1}\right\} \notin \operatorname{fil}\left(G_{0}^{*}\right)$.

It should be clear that the construction of $\bar{\alpha}^{n}$ 's is possible (remember that $\operatorname{fil}\left(G_{0}^{*}\right)$ is a weakly reasonable ultrafilter; use 1.5$)$. Let $C_{\omega}=\bigcap_{n<\omega} C_{n}$ and let $\left\langle\alpha_{\xi}^{\omega}: \xi<\lambda\right\rangle$ be the increasing enumeration of $C_{\omega}$. It follows from (ii) that for every $\varepsilon<\lambda$ the sequence $\left\langle\alpha_{\varepsilon+1}^{n}: n<\omega\right\rangle$ is strictly increasing and $\sup \left(\alpha_{\varepsilon+1}^{n}: n<\omega\right) \in C_{\omega}$, and if $\xi=\alpha_{\varepsilon}^{\omega}$, then $\sup \left(\alpha_{\xi+1}^{n}: n<\omega\right)=\alpha_{\varepsilon+1}^{\omega}$. It follows from (iii) that for every $n<\omega$,

$$
A_{n}:=\bigcup\left\{\left[\alpha_{\varepsilon}^{\omega}, \alpha_{\xi+1}^{n}\right): \varepsilon<\lambda \& \xi=\alpha_{\varepsilon}^{\omega}\right\} \notin \operatorname{fil}\left(G_{0}^{*}\right) .
$$

Fix $p \in G_{0}^{*}$ for a moment. By the choice of $\bar{\alpha}^{0}$ we know that the set $\left\{\xi<\lambda: f_{p}\left(\alpha_{\xi}^{0}\right)<\alpha_{\xi+1}^{0}\right\}$ is unbounded in $\lambda$, and hence also the set $\{\varepsilon<\lambda$ : $\left.f_{p}\left(\alpha_{\varepsilon}^{\omega}\right)<\alpha_{\varepsilon+1}^{\omega}\right\}$ is unbounded in $\lambda$. Therefore for some $n<\omega$ we have

$$
\left\|\left\{\varepsilon<\lambda: \xi=\alpha_{\varepsilon}^{\omega} \Rightarrow f_{p}\left(\alpha_{\varepsilon}^{\omega}\right)<\alpha_{\xi+1}^{n}\right\}\right\|=\lambda
$$

let $n(p)$ be the first such $n<\omega$. 
Note that if $p \leq^{0} q$ are from $G_{0}^{*}$, then $n(p) \leq n(q)$ (as $f_{p} \leq^{*} f_{q}$ ). Consequently, since $G_{0}^{*}$ is $\left(<\aleph_{1}\right)$-directed, there is $n^{*}<\omega$ such that $\left(\forall p \in G_{0}^{*}\right)$ $\left(n(p) \leq n^{*}\right)$. Look at the set $A_{n^{*}}$ : for every $p \in G_{0}^{*}$ there are $\lambda$ many $\varepsilon<\lambda$ such that $\alpha_{\varepsilon}^{\omega}<f_{p}\left(\alpha_{\varepsilon}^{\omega}\right)<\alpha_{\xi+1}^{n^{*}}$, where $\xi=\alpha_{\varepsilon}^{\omega}$, and so (by the definition of $f_{p}$ ) we get $A_{n^{*}} \in(\operatorname{fil}(p))^{+}$. Since $\operatorname{fil}\left(G_{0}^{*}\right)$ is an ultrafilter we get an immediate contradiction with $A_{n^{*}} \notin \operatorname{fil}\left(G_{0}^{*}\right)$.

(2) Suppose towards a contradiction that $\mathcal{F}_{1}$ is not club-dominating in ${ }^{\lambda} \lambda$. Then we may find an increasing function $h \in{ }^{\lambda} \lambda$ such that

$$
\left(\forall p \in G_{1}^{*}\right)\left(\left\{\varepsilon<\lambda: f_{p}(\varepsilon)<h(\varepsilon)\right\} \text { is stationary in } \lambda\right) .
$$

Pick an increasing continuous sequence $\left\langle\delta_{\xi}: \xi<\lambda\right\rangle \subseteq \lambda$ such that $(\forall \xi<\lambda)$ $\left(h\left(\delta_{\xi}\right)<\delta_{\xi+1}\right)$. Since $\operatorname{fil}\left(G_{1}^{*}\right)$ is weakly reasonable, we may use 1.5 to pick a club $C$ of $\lambda$ such that $C \subseteq\left\{\xi<\lambda: \delta_{\xi}=\xi\right.$ is a limit ordinal $\}$ and

$$
\bigcup\left\{\left[\delta_{\xi}, \delta_{\xi+1}\right): \xi \in C\right\} \notin \operatorname{fil}\left(G_{1}^{*}\right) .
$$

Since $\operatorname{fil}\left(G_{1}^{*}\right)$ is an ultrafilter, for some $p \in G_{1}^{*}$ we have

$$
\lambda \backslash \bigcup\left\{\left[\delta_{\xi}, \delta_{\xi+1}\right): \xi \in C\right\} \in \operatorname{fil}(p) .
$$

However, by the choice of $h$, the set $\left\{\xi<\lambda: \delta_{\xi}=\xi \in C \& f_{p}(\xi)<h(\xi)<\right.$ $\left.\delta_{\xi+1}\right\}$ is stationary (so of size $\lambda$ ), and we get an immediate contradiction with the definition of $f_{p}$.

Corollary 3.2 .

(1) If $G_{0}^{*} \subseteq \mathbb{Q}_{\lambda}^{0}$ is $\left(<\aleph_{1}\right)$-directed (with respect to $\leq^{0}$ ) and $\operatorname{fil}\left(G^{*}\right)$ is a weakly reasonable ultrafilter, then $\left\|G_{0}^{*}\right\| \geq \mathfrak{d}_{\lambda}$.

(2) If $G_{1}^{*} \subseteq \mathbb{Q}_{\lambda}^{0}$ is directed (with respect to $\leq^{0}$ ) and $\operatorname{fil}\left(G_{1}^{*}\right)$ is a weakly reasonable ultrafilter on $\lambda$, then $\left\|G_{1}^{*}\right\| \geq \mathfrak{d}_{\mathrm{cl}}(\lambda)$.

Proposition 3.3. Suppose that $G_{0}^{*} \subseteq \mathbb{Q}_{\lambda}^{0}$ is $(<\lambda)$-directed (with respect to $\left.\leq_{\mathbb{Q}_{\lambda}^{0}}\right)$ and $\operatorname{fil}\left(G^{*}\right)$ is an ultrafilter. For $p \in G_{0}^{*}$ let $f_{p} \in{ }^{\lambda} \lambda$ be defined as in 3.1. If $\mathcal{F}_{0}=\left\{f_{p}: p \in G_{0}^{*}\right\}$ is not a dominating family in ${ }^{\lambda} \lambda$, then $\lambda$ is measurable.

Proof. Similarly to the proof of $3.1(1)$, we note that $\mathcal{F}_{0}$ is $(<\lambda)$-directed (with respect to $\leq^{*}$ ). Assume $\mathcal{F}_{0}$ is not dominating family. Then we may choose an increasing continuous sequence $\left\langle\alpha_{\xi}: \xi<\lambda\right\rangle$ such that

$$
\left(\forall p \in G_{0}^{*}\right)\left(\exists^{\lambda} \varepsilon<\lambda\right)\left(f_{p}\left(\alpha_{\varepsilon}\right)<\alpha_{\varepsilon+1}\right) .
$$

Let

$$
\mathcal{U}=\left\{A \subseteq \lambda:\left(\exists p \in G_{0}^{*}\right)(\exists \delta<\lambda)(\forall \varepsilon>\delta)\left(f_{p}\left(\alpha_{\varepsilon}\right)<\alpha_{\varepsilon+1} \Rightarrow \varepsilon \in A\right)\right\} .
$$

We are going to show that $\mathcal{U}$ is a $\lambda$-complete uniform ultrafilter on $\lambda$. It should be clear that $\mathcal{U}$ includes all co-bounded subsets of $\lambda$ and that it is a 
$\lambda$-complete filter (remember that $\mathcal{F}_{0}$ is $(<\lambda)$-directed). To show that it is an ultrafilter suppose that $A \subseteq \lambda$ and let

$$
B=\bigcup\left\{\left[\alpha_{\varepsilon}, \alpha_{\varepsilon+1}\right): \varepsilon \in A\right\} \subseteq \lambda .
$$

Since $\operatorname{fil}\left(G_{0}^{*}\right)$ is an ultrafilter, then either $B \in \operatorname{fil}\left(G_{0}^{*}\right)$ or $\lambda \backslash B \in \operatorname{fil}\left(G_{0}^{*}\right)$. Suppose that the former happens, so we may choose $p \in G_{0}^{*}$ such that $B \in \operatorname{fil}(p)$. Then for some $\delta<\lambda$ we have

$$
\left(\forall \beta \in C^{p} \backslash \delta\right)\left(B \cap Z_{\beta}^{p} \in d_{\beta}^{p}\right) .
$$

Now, if $\varepsilon>\delta$ and $f_{p}\left(\alpha_{\varepsilon}\right)<\alpha_{\varepsilon+1}$, then $\left[\alpha_{\varepsilon}, \alpha_{\varepsilon+1}\right) \cap B \neq \emptyset$ and thus $\left[\alpha_{\varepsilon}, \alpha_{\varepsilon+1}\right) \subseteq B$, so $\varepsilon \in A$. Consequently, $A \in \mathcal{U}$ (as witnessed by $p, \delta$ ). In the same manner one shows that if $\lambda \backslash B \in \operatorname{fil}\left(G_{0}^{*}\right)$, then $\lambda \backslash A \in \mathcal{U}$.

4. Open problems and further investigations. It may well be that our forcing techniques for uncountable $\lambda$ are still not strong enough to carry out the arguments parallel to the consistency results for ultrafilters on $\omega$. However, we feel that the recent progress in the theory of forcing iterated with uncountable supports (as exemplified by [15], Rosłanowski and Shelah [12], [10], [9] and Eisworth [3]) may prove to be useful in developing iterated forcing for "killing" and/or "preserving" some subfamilies of the class of reasonable ultrafilters. In particular, in Rosłanowski and Shelah [8] we continue the research of the present paper and we introduce super reasonable ultrafilters which are stronger than very reasonable ultrafilters. We show that for inaccessible $\lambda$ it is consistent that there are such ultrafilters determined by generating systems of size less than $2^{\lambda}$, and we also prove a result on preserving them in $\lambda$-support iterations. We also show that consistently there are no ultrafilters generated by small systems. These results may be interpreted as some progress towards generalizing (a), (b) and (c) from the introduction. However, several other natural problems remain untouched. One of the main questions we are interested in are

Problem 4.1. Let $\lambda$ be a regular uncountable cardinal. Is it provable in $\mathrm{ZFC}$ that there exist reasonable ultrafilters on $\lambda$ ? Very reasonable? (See $2.5(4,5)$.)

PROBLEM 4.2. Is it consistent that there exists a very reasonable ultrafilter $D$ on $\lambda$ such that for every very reasonable ultrafilter $D^{\prime}$ on $\lambda$ for some function $f \in \mathcal{F}_{\lambda}$ we have $D / f=D^{\prime} / f$ ?

Since in the present paper we deal with dividing by $f \in \mathcal{F}_{\lambda}$, and the normal ultrafilters are fixed points for this operation, the natural question is: 
Problem 4.3. Is it consistent that for every $D \in$ uuf there is $f \in \mathcal{F}_{\lambda}$ such that either $D / f$ is normal or $D / f$ is reasonable (or even very reasonable)?

We may also re-interpret our aim as follows.

DEFINITION 4.4.

(1) Let $\mathrm{UE}_{\lambda, \mu}^{*}$ be the family of all $(<\mu)$-directed (with respect to $\leq^{0}$ ) subsets $G^{*}$ of $\mathbb{Q}_{\lambda}^{0}$ such that $\operatorname{fil}\left(G^{*}\right)$ is a proper ultrafilter on $\lambda$.

(2) $\mathrm{UF}_{\lambda, \mu}^{*}=\left\{\operatorname{fil}\left(G^{*}\right): G^{*} \in \mathrm{UE}_{\lambda, \mu}^{*}\right\} ; \mathrm{UF}_{\lambda}^{*}=\mathrm{UF}_{\lambda, \lambda^{+}}^{*}$ and $\mathrm{UF}_{\lambda}=\mathrm{UF}_{\lambda, \aleph_{0}}^{*}$.

AIM 4.5. Investigate $\mathrm{UF}_{\lambda}^{*}, \mathrm{UF}_{\lambda}$; in particular check if any two of them can have common quotients.

We expect that the forcing theorems needed for further research will be similar to [15] and even more so to [12, 10, 9], in some respects, and for others to [13].

Let us also note that combinatorial aspects of generating ultrafilters by means similar to generating systems of Definition 2.5 will be studied in Rosłanowski and Shelah [7].

\section{References}

[1] A. Blass and S. Shelah, There may be simple $P_{\aleph_{1}}$ - and $P_{\aleph_{2}}$-points and the RudinKeisler ordering may be downward directed, Ann. Pure Appl. Logic 33 (1987), 213243.

[2] J. Cummings and S. Shelah. Cardinal invariants above the continuum, ibid. 75 (1995), 251-268.

[3] T. Eisworth, On iterated forcing for successors of regular cardinals, Fund. Math. 179 (2003), 249-266.

[4] M. Gitik, On non-minimal p-points over a measurable cardinal, Ann. Math. Logic 20 (1981), 269-288.

[5] T. Jech, Set Theory, Academic Press, New York, 1978.

[6] P. Matet, A. Rosłanowski, and S. Shelah, Cofinality of the non-stationary ideal, Trans. Amer. Math. Soc. 357 (2005), 4813-4837.

[7] A. Rosłanowski and S. Shelah, Generating ultrafilters in a reasonable way, preprint, math.LO/0607218.

[8] —, - Reasonable ultrafilters, again, preprint. math.LO/0605067.

[9] - - - Reasonably complete forcing notions, Quaderni Mat., to appear; math.LO/ 0508272 .

[10] - - - Sheva-Sheva-Sheva: large creatures, Israel J. Math., to appear; math.LO/ 0210205.

[11] -, - Norms on possibilities I: forcing with trees and creatures, Mem. Amer. Math. Soc. 141 (1999), no. 671.

[12] -, - Iteration of $\lambda$-complete forcing notions not collapsing $\lambda^{+}$, Internat. J. Math. Math. Sci. 28 (2001), 63-82.

[13] S. Shelah, Incompactness in singular, preprint.

[14] -, Proper and Improper Forcing, Perspectives in Math. Logic, Springer, 1998. 
[15] S. Shelah, Not collapsing cardinals $\leq \kappa$ in $(<\kappa)$-support iterations, Israel J. Math. 136 (2003) 29-115.

Einstein Institute of Mathematics

Edmond J. Safra Campus, Givat Ram

The Hebrew University of Jerusalem

Jerusalem, 91904, Israel

E-mail: shelah@math.huji.ac.il

URL: http://shelah.logic.at
Department of Mathematics

Rutgers University

New Brunswick, NJ 08854, U.S.A.

Received 10 August 2004;

in revised form 15 May 2006 